TRANSACTIONS OF THE

AMERICAN MATHEMATICAL SOCIETY

Volume 349, Number 7, July 1997, Pages 2917-2940

S $0002-9947(97) 01474-8$

\title{
THE LOCAL DIMENSIONS OF THE BERNOULLI CONVOLUTION ASSOCIATED WITH THE GOLDEN NUMBER
}

\author{
TIAN-YOU HU
}

Abstract. Let $X_{1}, X_{2}, \ldots$ be a sequence of i.i.d. random variables each taking values of 1 and -1 with equal probability. For $1 / 2<\rho<1$ satisfying the equation $1-\rho-\cdots-\rho^{s}=0$, let $\mu$ be the probability measure induced by $S=\sum_{i=1}^{\infty} \rho^{i} X_{i}$. For any $x$ in the range of $S$, let

$$
d(\mu, x)=\lim _{r \rightarrow 0^{+}} \log \mu([x-r, x+r]) / \log r
$$

be the local dimension of $\mu$ at $x$ whenever the limit exists. We prove that

$$
\alpha^{*}=-\frac{\log 2}{\log \rho} \quad \text { and } \quad \alpha_{*}=-\frac{\log \delta}{s \log \rho}-\frac{\log 2}{\log \rho},
$$

where $\delta=(\sqrt{5}-1) / 2$, are respectively the maximum and minimum values of the local dimensions. If $s=2$, then $\rho$ is the golden number, and the approximate numerical values are $\alpha^{*} \approx 1.4404$ and $\alpha_{*} \approx 0.9404$.

\section{INTRODUCTION}

Let $X_{1}, X_{2}, \ldots$ be a sequence of i.i.d. random variables which take values 1 and -1 with equal probability. For $0<\rho<1$, let $\mu$ be the infinitely convolved Bernoulli measure induced by

$$
S=\sum_{i=1}^{\infty} \rho^{i} X_{i}
$$

The measure $\mu$ has been studied for over half a century but is only partially understood today. It follows from a theorem of Jessen and Wintner [JW] that $\mu$ is either absolutely continuous or purely singular. Erdös proved that $\mu$ is absolutely continuous for almost all $\rho$ close enough to one $[\mathrm{E}]$. He conjectured that the result should be true for almost all $1 / 2<\rho<1$. Solomyak [So] has recently proved this conjecture to be true. In spite of this, the only explicit values of $\rho$ for which $\mu$ is known to be absolutely continuous are $\rho=2^{-1 / n}, n=1,2, \ldots$, and a family of algebraic numbers discovered by A. Garsia [G]. On the other hand, if $\rho^{-1}$ is a Pisot-Vijayaraghavan (PV)-number (recall that an algebraic integer $\alpha>1$ is called a PV-number if all its conjugates are in absolute value less than one), then $\mu$ is purely singular, and the numbers of this class are the only numbers (for $\rho>1 / 2$ )

Received by the editors August 23, 1994 and, in revised form, January 25, 1995.

1991 Mathematics Subject Classification. Primary 28A80; Secondary 42A85.

Key words and phrases. Bernoulli convolution, Fibonacci sequence, local dimension, PVnumber. 
for which $\mu$ is known to be purely singular. Examples of such $\rho$ are the roots of the polynomials

$$
1-x-x^{2}-\cdots-x^{s},
$$

$s=2,3, \ldots$ The smallest PV-number is the positive root of $x^{3}-x-1$ with $\rho^{-1} \approx 1.324718(\rho \approx 0.7548777)[\mathrm{Si}$ ]. The reader may refer to [Sa, BDGPS] for further information regarding the properties and applications of the PV-numbers.

The type of the measure $\mu$ will affect the Hausdorff dimension of the graph of $S$ when $X_{i}$ 's are the Rademacher functions on $[0,1]$. If $\mu$ is absolutely continuous with $d \mu / d x$ in $L^{p}$ for some $p>1$, then the Hausdorff dimension of the graph of $S$ is $2+\frac{\log \rho}{\log 2}$ [HL1, PU] and the Hausdorff dimension of the level set is $1+\frac{\log \rho}{\log 2}$ a.e. [HL2]. If $\rho^{-1}$ is a PV-number, then the Hausdorff dimension of the graph is strictly less than $2+\frac{\log \rho}{\log 2}[\mathrm{PU}]$.

The degree of singularity of $\mu$ can be analyzed on a pointwise basis by studying its local dimensions and on an average by studying its entropy, information dimension, Hausdorff dimension or $L^{p}$-dimension. The upper local dimension of $\mu$ at $x$ is defined by

$$
\bar{d}(\mu, x)=\varlimsup_{r \rightarrow 0^{+}} \frac{\log \mu(I(x, r))}{\log r},
$$

where $I(x, r)$ is the closed interval $[x-r, x+r]$. Similarly the lower local dimension $\underline{d}(\mu, x)$ is defined by using the lower limit. When $\bar{d}(\mu, x)=\underline{d}(\mu, x)$, the common value is called the local dimension of $\mu$ at $x$ and is denoted by $d(\mu, x)$.

Note that $\mu$ is a self-similar measure satisfying the equation

$$
\mu=\frac{1}{2} \mu \circ w_{1}^{-1}+\frac{1}{2} \mu \circ w_{2}^{-1},
$$

where $w_{1}(x)=\rho x$ and $w_{2}(x)=\rho x+(1-\rho)[\mathrm{L} 1$, Theorem 4.3]. For $0<\rho<1 / 2$, $\mu$ is a singular Cantor-type measure satisfying the measure separation (i.e., the measures $\mu \circ w_{1}^{-1}$ and $\mu \circ w_{2}^{-1}$ are mutually singular) and the open set condition, so complete results on the local dimensions are known [GH]. If $1 / 2<\rho<1$, then $\mu$ does not satisfy either of the two separation conditions, so no previous result is available on the local dimensions.

Recently, several authors $[\mathrm{AY}, \mathrm{AZ}, \mathrm{LP}]$ have studied the entropy and the information dimension of $\mu$. For $\rho=(\sqrt{5}-1) / 2$, a rigorous estimate for the entropy of $\mu$ has been obtained [AZ], which agrees with [AY] numerical calculations; also an explicit theoretical formula for the information dimension and Hausdorff dimension of $\mu$ has been obtained [LP].

Lau [L1, L2] has succeeded in calculating the $L^{2}$-dimension of $\mu$ for which $\rho^{-1}$ is in the class of F-numbers including all PV-numbers. Recall [St] that the $L^{p_{-}}$ dimension of $\mu$ is defined by

$$
\operatorname{dim}_{p}(\mu)=\lim _{r \rightarrow 0^{+}} \frac{\log \sup \sum_{j} \mu\left(A_{j}\right)^{p}}{(p-1) \log r},
$$

provided the limit exists, where the supremum is over all partitions of $\mathbb{R}$ into sets $A_{j}$ of diameter $\leq r$. In particular, if $\rho$ is the golden number, then the $L^{2}$-dimension is $\alpha=0.9923995 \cdots$; this number is given by the equation $\left(4 \rho^{\alpha}\right)^{3}-2\left(4 \rho^{\alpha}\right)^{2}-$ $2\left(4 \rho^{\alpha}\right)+2=0[\mathrm{~L} 1]$. This result has now been generalized in a more recent joint work of Lau and Ngai [LN1, LN2], where the $L^{p}$-dimension of $\mu$, for every integer $p>1$, is calculated and the multifractal structure of $\mu$ is analyzed. 
In this paper we will give a detailed study of the local dimensions of $\mu$ for $\rho=(\sqrt{5}-1) / 2$. The results can be easily generalized to the case in which $\rho$ is a root of (1.1). To find the extreme values of the local dimensions, we actually go through the computation for the $\alpha$-density of $\mu$. Recall [F] that the $\alpha$-upper and the $\alpha$-lower density of $\mu$ at $x$ are defined by $\varlimsup_{r \rightarrow 0^{+}} \mu(I(x, r)) / r^{\alpha}$ and $\underline{\lim }_{r \rightarrow 0^{+}} \mu(I(x, r)) / r^{\alpha}$, respectively. If both limits are positive, it is clear that $d(\mu, x)=\alpha$. The converse, however, is not true.

For $1 / 2 \leq \rho<1$, the range of $S$ is the interval $[-\ell, \ell]$, where $\ell=\sum_{i=1}^{\infty} \rho^{i}=$ $\rho /(1-\rho)$. For $x=\sum_{i=1}^{\infty} \varepsilon_{i} \rho^{i}$ for some sequence $\varepsilon=\left(\varepsilon_{1}, \varepsilon_{2}, \ldots\right) \in\{-1,1\}^{\infty}$, let $E(x)$ be the set consisting of all points in $[-\ell, \ell]$ having the same "tail coefficients $\varepsilon_{i}$, for $i \geq N "$ as $x$. More precisely, let

$$
E(x)=\bigcup_{n=0}^{\infty}\left\{ \pm \rho^{n} x+\sum_{i=0}^{n} \tau_{i} \rho^{i}: \tau_{0}=0 \text { and } \tau_{i}= \pm 1 \text { for } i=1,2, \ldots, n\right\}
$$

Obviously, $\pm x \in E(x)$ and $E(x)$ is dense in $[-\ell, \ell]$. The main results we will prove are the following:

Theorem A. (i) Let $1 / 2<\rho<1$. Then $\alpha^{*}=-\frac{\log 2}{\log \rho}$ is the maximum value of $\bar{d}(\mu, x)$ for all $x \in[-\ell, \ell]$; furthermore,

$$
0<\varliminf_{r \rightarrow 0^{+}} \mu(I(z, r)) / r^{\alpha^{*}} \leq \varlimsup_{r \rightarrow 0^{+}} \mu(I(z, r)) / r^{\alpha^{*}}<\infty
$$

for $z=\ell($ or $-\ell)$.

(ii) If $\rho$ is a root of (1.1), then $\alpha_{*}=-\frac{\log \delta}{s \log \rho}-\frac{\log 2}{\log \rho}$, where $\delta=(\sqrt{5}-1) / 2$, is the minimum value of $\underline{d}(\mu, x)$ for all $x \in[-\ell, \ell]$; furthermore,

$$
0<\varliminf_{r \rightarrow 0^{+}} \mu(I(z, r)) / r^{\alpha_{*}} \leq \varlimsup_{r \rightarrow 0^{+}} \mu(I(z, r)) / r^{\alpha_{*}}<\infty
$$

for every point $z$ in the dense subset $E\left(\left(\rho^{s+2}+\rho^{s+3}+\cdots+\rho^{2 s}\right) /\left(1-\rho^{2 s}\right)\right)$ of $[-\ell, \ell]$.

Theorem B. Suppose that $\rho$ is a root of (1.1). Then

(i) For every $\alpha_{*} \leq \alpha \leq \alpha^{*}$, there exists a point $z(\alpha)$ such that $d(\mu, z)=\alpha$ for every point $z$ in the dense subset $E(z(\alpha))$ of $[-\ell, \ell]$;

(ii) for any $\alpha_{*}<\alpha_{1}<\alpha_{2}<\alpha^{*}$, there exists a point $z\left(\alpha_{1}, \alpha_{2}\right)$ such that $\underline{d}(\mu, z)<$ $\alpha_{1}<\alpha_{2}<\bar{d}(\mu, z)$ for every point $z$ in the dense subset $E\left(z\left(\alpha_{1}, \alpha_{2}\right)\right)$ of $[-\ell, \ell]$.

Observe that for roots of (1.1), $\rho \rightarrow 1 / 2$ from above as $s \rightarrow \infty$. So both $\alpha^{*}$ and $\alpha_{*}$ approach 1 . Also $\mu$ is uniformly distributed for $\rho=1 / 2$; in this case, $d(\mu, x)=1$ for every $x \in[-\ell, \ell]$. If $s=2$, then $\rho=(\sqrt{5}-1) / 2$, and the approximate numerical values are $\alpha^{*} \approx 1.4404$ and $\alpha_{*} \approx 0.9404$. The picture of the measure $\mu$ associated with $\rho=(\sqrt{5}-1) / 2$ is shown in Figure 1 . The horizontal axis represents the support of the measure. The vertical axis depicts the weight of the measure over small intervals divided by the length of the interval. The minimum value of $\underline{d}(\mu, x)$ occurs at every point in the dense subset $E\left(\rho^{4} /\left(1-\rho^{4}\right)\right)$. The two points $\pm z= \pm \rho^{4} /\left(1-\rho^{4}\right)$ have been indicated in the figure.

The ideas for the proofs of Theorems A and B are as follows. Let $\mu_{n}$ be the probability measure induced by the partial sum

$$
S_{n}=\sum_{i=1}^{n} \rho^{i} X_{i}
$$




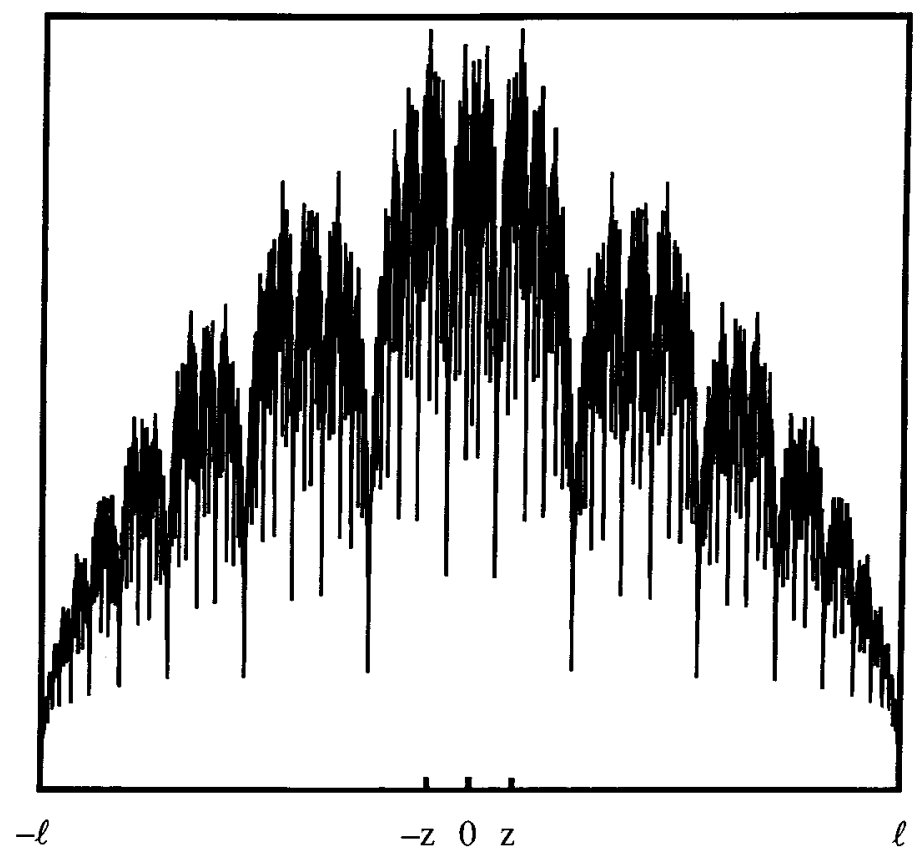

Figure 1. A histogram of the Bernoulli measure associated with the golden number $\rho=(\sqrt{5}-1) / 2$. The minimum value of the local dimensions occurs at $\pm z$. The maximum value of the local dimensions occurs at $\pm \ell$.

For any value $v$ of $S_{n}$, let

$$
\langle v\rangle=\left\{\left(\varepsilon_{1}, \ldots, \varepsilon_{n}\right) \in\{-1,1\}^{n}: \sum_{i=1}^{n} \varepsilon_{i} \rho^{i}=v\right\},
$$

and let $\#\langle v\rangle$ be the cardinality of $\langle v\rangle$. Since the $X_{i}$ 's are independent, we have

$$
\mu_{n}(\{v\})=2^{-n} \#\langle v\rangle .
$$

For any $x \in[-\ell, \ell]$, we have $x=\sum_{i=1}^{\infty} \varepsilon_{i} \rho^{i}$ for some sequence $\varepsilon=\left(\varepsilon_{1}, \varepsilon_{2}, \ldots\right) \in$ $\{-1,1\}^{\infty}$ (in general this representation is not unique for $1 / 2<\rho<1$ ). Let $x_{n}=\sum_{i=1}^{n} \varepsilon_{i} \rho^{i}$ be the $n$th partial sum. Under some conditions, $\mu(I(x, r))$ can be estimated by using $\mu_{n}\left(\left\{x_{n}\right\}\right)=2^{-n} \#\left\langle x_{n}\right\rangle$. Thus the key is to determine the rate of growth of \# $\left\langle x_{n}\right\rangle$.

Observe that if $\left(\tau_{1}, \ldots, \tau_{n}\right)$ and $\left(\omega_{1}, \ldots, \omega_{n}\right)$ are two points in $\left\langle x_{n}\right\rangle$, then $\sum_{\text {let }}^{n}\left(\tau_{i}-\omega_{i}\right) \rho^{i}=0$. Hence we introduce the notion of a zero element. For each $n$,

$$
\mathbb{Z}_{n}=\left\{\left(a_{1}, \ldots, a_{n}\right) \in\{-1,0,1\}^{n}: \sum_{i=1}^{n} a_{i} \rho^{i}=0\right\} .
$$

Members of $\mathbb{Z}_{n}$ are called zero elements (of length $n$ ). For the coefficients $\varepsilon^{(\mathbf{n})}=$ $\left(\varepsilon_{1}, \ldots, \varepsilon_{n}\right)$ of $x_{n}$, let $p_{n}=\#\left\{\left(a_{1}, \ldots, a_{n}\right) \in \mathbb{Z}_{n}: a_{i}=\varepsilon_{i}\right.$ for $i=1, \ldots, n$ for which $\left.a_{i} \neq 0\right\}$, i.e., $p_{n}$ is the cardinality of the subset of $\mathbb{Z}_{n}$ consisting of those zero elements contained in $\varepsilon^{(\mathbf{n})}$. It can be shown that $p_{n}=\#\left\langle x_{n}\right\rangle$. To calculate this 
number, we decompose a zero element into a direct sum of atoms (defined in $\S 3$ ), and derive a recurrence equation for computing $p_{n}$ based on some atoms contained in $\varepsilon^{(\mathbf{n})}$. It is an interesting discovery that for the points where the minimum value of the local dimension occurs, the rate of growth of $p_{n}$ is comparable to that of the Fibonacci sequence.

The paper is organized as follows: In $\S 2$ we derive some general lemmas which will reduce the computation for $d(\mu, x)$ to the computation of \# $\left\langle x_{n}\right\rangle$. Part (i) of Theorem A (Theorem 2.3) is also proved in this section. In $\S 3$, we study the structure of zero elements and give decomposition theorems for zero elements (Theorems 3.2, 3.7) and a recurrence equation for computing $p_{n}$ (Lemma 3.5). In particular, when $\rho$ is a root of (1.1), concrete forms of zero elements are given. Part (ii) of Theorem A and Theorem B are the main theorems of the paper. By using the ideas described above, proofs of these theorems will be established in $\S 4$ and $\S 5$ respectively.

Throughout we will use the notations introduced in $\S 1$. We will use $\varepsilon_{i}, \tau_{i}, \omega_{i}, \ldots$ to denote variables taking values in $\{1,-1\}$, and $a_{i}, b_{i}, c_{i}, \ldots$ to denote variables taking values in $\{1,0,-1\}$. In particular, if $x \in[-\ell, \ell]$ is associated with some series representation, then $x_{n}$ will mean the $n$th partial sum with respect to this representation.

\section{Some Lemmas}

Lemma 2.1. Let $0<\rho<1$. For any $\alpha \geq 0$ and for any $x \in[-\ell, \ell]$,

(i) $\varliminf_{r \rightarrow 0^{+}} \mu(I(x, r)) / r^{\alpha}>0$ if and only if $\underline{\lim }_{n \rightarrow \infty} \mu_{n}\left(I\left(x,(1+\ell) \rho^{n}\right)\right) / \rho^{n \alpha}>0$; and

(ii) $\varlimsup_{r \rightarrow 0^{+}} \mu(I(x, r)) / r^{\alpha}<\infty$ if and only if $\overline{\lim }_{n \rightarrow \infty} \mu_{n}\left(I\left(x,(1+\ell) \rho^{n}\right)\right) / \rho^{n \alpha}<$ $\infty$.

Proof. Since $\left|S_{n}-S\right| \leq \rho^{n+1}+\rho^{n+2}+\cdots=\ell \rho^{n}$, we have

$$
\begin{aligned}
\mu_{n}\left(I\left(x,(1+\ell) \rho^{n}\right)\right) & =\operatorname{Prob}\left(x-(1+\ell) \rho^{n}<S_{n} \leq x+(1+\ell) \rho^{n}\right) \\
& \leq \operatorname{Prob}\left(x-(1+\ell) \rho^{n}-\ell \rho^{n}<S \leq x+(1+\ell) \rho^{n}+\ell \rho^{n}\right) \\
& =\mu\left(I\left(x,(1+2 \ell) \rho^{n}\right)\right) .
\end{aligned}
$$

Similarly

$$
\mu\left(I\left(x, \rho^{n}\right)\right) \leq \mu_{n}\left(I\left(x,(1+\ell) \rho^{n}\right)\right) .
$$

For any $r>0$, there exists some $n$ such that $(1+2 \ell) \rho^{n}<r \leq(1+2 \ell) \rho^{n-1}$. Using (2.1) we have

$$
\mu(I(x, r)) / r^{\alpha} \geq \mu\left(I\left(x,(1+2 \ell) \rho^{n}\right)\right) /\left[(1+2 \ell) \rho^{n-1}\right]^{\alpha} \geq \mu_{n}\left(I\left(x,(1+\ell) \rho^{n}\right)\right) / C \rho^{n \alpha},
$$

where $C=[(1+2 \ell) / \rho]^{\alpha}>0$. So $\varliminf_{n \rightarrow \infty} \mu_{n}\left(I\left(x,(1+\ell) \rho^{n}\right)\right) / \rho^{n \alpha}>0$ implies $\varliminf_{r \rightarrow 0^{+}} \mu(I(x, r)) / r^{\alpha}>0$. The converse can be proved similarly by using (2.2). Therefore (i) is true. The proof for (ii) is similar.

We omit the simple proof for the following lemma.

Lemma 2.2. Let $0<\rho<1$. For any $x \in[-\ell, \ell]$, then

$$
\bar{d}(\mu, x)=\varlimsup_{n \rightarrow \infty} \frac{\log \mu_{n}\left(I\left(x,(1+\ell) \rho^{n}\right)\right)}{n \log \rho} \text { and } \underline{d}(\mu, x)=\varliminf_{n \rightarrow \infty} \frac{\log \mu_{n}\left(I\left(x,(l+\ell) \rho^{n}\right)\right)}{n \log \rho} .
$$

According to Lemma 2.2, the maximum (minimum) value of the local dimension will occur at a point $x$ where $\mu_{n}\left(I\left(x,(1+\ell) \rho^{n}\right)\right)$ has a minimum (maximum) value. The first case occurs when $x$ is one of the end points of $[-\ell, \ell]$. 
Theorem 2.3. Let $0<\rho<1$. Then $\alpha^{*}=-\frac{\log 2}{\log \rho}$ is the maximum value of $\bar{d}(\mu, x)$ for all $x$. Furthermore,

$$
0<\varliminf_{r \rightarrow 0^{+}} \mu(I(z, r)) / r^{\alpha^{*}} \leq \varlimsup_{r \rightarrow 0^{+}} \mu(I(z, r)) / r^{\alpha^{*}}<\infty \quad \text { for } z=\ell(\text { or }-\ell) .
$$

Proof. Let $x \in[-\ell, \ell]$ be any point. Since $x_{n} \in I\left(x,(1+\ell) \rho^{n}\right)$ for all series representations of $x$ and for all $n$, by (1.3) we have

$$
\mu_{n}\left(I\left(x,(1+\ell) \rho^{n}\right)\right) \geq \mu_{n}\left(\left\{x_{n}\right\}\right)=2^{-n} \#\left\langle x_{n}\right\rangle .
$$

Hence from Lemma 2.2 we deduce that

$$
\bar{d}(\mu, x) \leq \varlimsup_{n \rightarrow \infty} \frac{\log 2^{-n}}{n \log \rho}=\alpha^{*} .
$$

Now let $z=\ell=\sum_{i=1}^{\infty} \rho^{i}$. Then $z_{n}=\sum_{i=1}^{n} \rho^{i}$ is the maximum value of $S_{n}$ and clearly $\#\left\langle z_{n}\right\rangle=1$. The one immediately smaller than $z_{n}$ is $z^{\prime}=\left(\sum_{i=1}^{n-1} \rho^{i}\right)-\rho^{n}$ and $z-z^{\prime}=(2+\ell) \rho^{n}$. So $I\left(z,(1+\ell) \rho^{n}\right)$ contains exactly one value of $S_{n}$, namely $z_{n}$, for all $n$. Therefore

$$
\mu_{n}\left(I\left(z,(1+\ell) \rho^{n}\right)\right)=2^{-n} \#\left\langle z_{n}\right\rangle=2^{-n} \quad \text { and } \quad \mu_{n}\left(I\left(z,(1+\ell) \rho^{n}\right)\right) / \rho^{n \alpha^{*}}=1
$$

for all $n$. So the second statement of the theorem follows from Lemma 2.1

The key idea used in the proof is that $\mu_{n}\left(I\left(z,(1+\ell) \rho^{n}\right)\right)$ can be estimated by $2^{-n} \#\left\langle z_{n}\right\rangle$; thus $d(\mu, z)$ can be computed by

$$
\lim _{n \rightarrow \infty} \frac{\log \left(2^{-n} \#\left\langle z_{n}\right\rangle\right)}{n \log \rho}=-\frac{\log 2}{\log \rho} .
$$

In the following we will impose some conditions on $S$ so that this idea will be still valid for computing the local dimension of $\mu$ at other points.

$S$ is said to satisfy condition A if there is a constant $C$ such that for any $n$, every interval of length $\rho^{n}$ contains at most $C$ distinct values of $S_{n}$. It is known that if $\rho^{-1}$ is a PV-number, then $S$ satisfies condition A ([G], Lemma 1.51). Let $x$ be associated with a series representation. By definition, either $x_{n+1}=x_{n}-\rho^{n+1}$ or $x_{n+1}=x_{n}+\rho^{n+1}$. In either case, there is a fixed $\varepsilon_{n+1}=1$ (or -1$)$ such that for every $\left(\varepsilon_{1}, \ldots, \varepsilon_{n}\right) \in\left\langle x_{n}\right\rangle$, we have $\left(\varepsilon_{1}, \ldots, \varepsilon_{n}, \varepsilon_{n+1}\right) \in\left\langle x_{n+1}\right\rangle$. So

$$
\#\left\langle x_{n+1}\right\rangle \geq \#\left\langle x_{n}\right\rangle \text {. }
$$

For any $n$, let $V_{n}$ be the set consisting of all distinct values of $S_{n}$.

Lemma 2.4. Let $0<\rho<1$. Suppose that $S$ satisfies condition A. Assume that there exist a series representation $z=\sum_{i=1}^{\infty} \varepsilon_{i} \rho^{i}$, a constant $C$ and a sequence of positive integers $n_{1}<n_{2}<\cdots$ with the following three properties:

(i) $n_{k}-n_{k-1} \leq C$ for all $k$;

(ii) $C \#\left\langle z_{n_{k}}\right\rangle \geq \#\langle v\rangle$ for all $k$ and for all $v \in V_{n_{k}}$; and

(iii) $0<\underline{\lim }_{k \rightarrow \infty} \#\left\langle z_{n_{k}}\right\rangle /\left(2 \rho^{\alpha_{*}}\right)^{n_{k}} \leq \varlimsup_{k \rightarrow \infty} \#\left\langle z_{n_{k}}\right\rangle /\left(2 \rho^{\alpha_{*}}\right)^{n_{k}}<\infty$, for some $\alpha_{*} \geq 0$.

Then

(a) $\alpha_{*}=\lim _{k \rightarrow \infty}\left(\log \#\left\langle z_{n_{k}}\right\rangle / n_{k} \log \rho\right)-\log 2 / \log \rho$;

(b) $0<\varliminf_{r \rightarrow 0^{+}} \mu(I(z, r)) / r^{\alpha_{*}} \leq \lim _{r \rightarrow 0^{+}} \mu(I(z, r)) / r^{\alpha_{*}}<\infty$, hence $d(\mu, z)=$ $\alpha_{*} ;$ and

(c) $\alpha_{*}$ is the minimum value of $\underline{d}(\mu, x)$ for all $x$. 
Proof. By property (iii), for all large $k$, we have

$$
0<N<\#\left\langle z_{n_{k}}\right\rangle /\left(2 \rho^{\alpha_{*}}\right)^{n_{k}}<M<\infty .
$$

By taking the logarithm, followed by dividing by $n_{k} \log \rho$ on both sides, we obtain

$$
\frac{\log N}{n_{k} \log \rho}>\frac{\log \#\left\langle z_{n_{k}}\right\rangle}{n_{k} \log \rho}-\frac{\log 2}{\log \rho}-\alpha_{*}>\frac{\log M}{n_{k} \log \rho} .
$$

Thus (a) follows by letting $k \rightarrow \infty$.

Now for any integer $n$, there exists $k$ such that $n_{k-1}<n \leq n_{k}$. Using (1.3) and condition A, we obtain

$$
\begin{array}{rlrl}
\mu_{n}\left(I\left(z,(1+\ell) \rho^{n}\right)\right) & =2^{-n} \sum\left\{\#\langle v\rangle: v \in I\left(z,(1+\ell) \rho^{n}\right) \cap V_{n}\right\} \\
& \leq C_{1} 2^{-n} \max \left\{\#\langle v\rangle: v \in I\left(z,(1+\ell) \rho^{n}\right) \cap V_{n}\right\} \\
& \leq C_{1} 2^{-n} \max \left\{\#\langle v\rangle: v \in V_{n_{k}}\right\} & & \text { (by (2.4)) } \\
& \leq C_{2} 2^{-n_{k}} \#\left\langle z_{n_{k}}\right\rangle 2^{n_{k}-n} & & \text { (by property (ii)) } \\
& \leq C_{3} 2^{-n_{k}} \#\left\langle z_{n_{k}}\right\rangle & & \text { (by property (i)). }
\end{array}
$$

Similarly, by using (2.3), we have

$$
\mu_{n}\left(I\left(z,(1+\ell) \rho^{n}\right)\right) \geq 2^{-n} \#\left\langle z_{n}\right\rangle \geq C_{4} 2^{-n_{k-1}} \#\left\langle z_{n_{k-1}}\right\rangle .
$$

Applying property (i) again, we obtain

$$
C_{5} \#\left\langle z_{n_{k-1}}\right\rangle /\left(2 \rho^{\alpha_{*}}\right)^{n_{k-1}} \leq \mu_{n}\left(I\left(z,(1+\ell) \rho^{n}\right)\right) / \rho^{\alpha_{*} n} \leq C_{6} \#\left\langle z_{n_{k}}\right\rangle /\left(2 \rho^{\alpha_{*}}\right)^{n_{k}} .
$$

The constants $C_{i}$, for $i=1, \ldots, 6$, are independent of $n$. So (b) follows from this estimate, property (iii) and Lemma 2.1. To show (c), for any $x \in[-\ell, \ell]$ and for any $n$, in a manner similar to $(2.5)$, we have for $n \leq n_{k}$

$$
\mu_{n}\left(I\left(x,(1+\ell) \rho^{n}\right)\right) \leq C_{3} 2^{-n_{k}} \#\left\langle z_{n_{k}}\right\rangle \leq C_{3} \mu_{n_{k}}\left(I\left(z,(1+\ell) \rho^{n}\right)\right) .
$$

Hence Lemma 2.2 implies $\underline{d}(\mu, x) \geq \underline{d}(\mu, z)=\alpha_{*}$.

An immediate application of Lemma 2.4 is the following computation of the $\alpha$-density of $\mu$ for $0<\rho \leq 1 / 2$. This result was previously known.

Corollary 2.5. Let $0<\rho \leq 1 / 2$, and let $\alpha=-\frac{\log 2}{\log \rho}$. Then for every value $z$ of $S$, we have $0<\varliminf_{r \rightarrow 0^{+}} \mu(I(z, r)) / r^{\alpha} \leq \varlimsup_{r \rightarrow 0^{+}} \mu(I(z, r)) / r^{\alpha}<\infty$; hence $d(\mu, z)=\alpha$.

Proof. For $0<\rho \leq 1 / 2$, it is easy to verify that there is a constant $C>0$ such that $\left|v-v^{\prime}\right| \geq C \rho^{n}$ for all $n$ and for all $v, v^{\prime}$ in $V_{n}$. Therefore $S$ satisfies condition A.

Let $z$ be any value of $S$ with a series representation. Then $\#\left\langle z_{n}\right\rangle=1$ for all $n$. It follows that $\#\left\langle z_{n}\right\rangle /\left(2 \rho^{\alpha}\right)^{n}=1$ for all $n$. Thus $z$ and the integer sequence $1,2, \ldots$ satisfy (i), (ii) and (iii) in Lemma 2.4 , so $0<\varliminf_{r \rightarrow 0^{+}} \mu(I(z, r)) / r^{\alpha} \leq$ $\varlimsup_{r \rightarrow 0^{+}} \mu(I(z, r)) / r^{\alpha}<\infty$.

For $1 / 2<\rho<1$, in order to calculate the local dimension at any point, we impose another condition on $S$. Let $x_{n}(1)<x_{n}(2)<\cdots<x_{n}\left(i_{n}\right)$, where $1 \leq i_{n} \leq 2^{n}$, be all distinct values of $S_{n}$. $S$ is said to satisfy condition B if there is a sequence $\theta_{1}, \theta_{2}, \ldots$ with $\lim _{n \rightarrow \infty}\left(\log \theta_{n} / n\right)=0$ such that $1 / \theta_{n} \leq \#\left\langle x_{n}(j+1)\right\rangle / \#\left\langle x_{n}(j)\right\rangle \leq$ $\theta_{n}$ for all $n$ and for all $1 \leq j<i_{n}$. 
Lemma 2.6. Let $1 / 2<\rho<1$. If $S$ satisfies both condition A and condition B, then for any $x \in[-\ell, \ell]$ and for any series representation $x=\sum_{i=1}^{\infty} \varepsilon_{i} \rho^{i}$, we have

$$
\bar{d}(\mu, x)=\varlimsup_{n \rightarrow \infty} \frac{\log \#\left\langle x_{n}\right\rangle}{n \log \rho}-\frac{\log 2}{\log \rho} \quad \text { and } \quad \underline{d}(\mu, x)=\varliminf_{n \rightarrow \infty} \frac{\log \#\left\langle x_{n}\right\rangle}{n \log \rho}-\frac{\log 2}{\log \rho} .
$$

Proof. We first prove that the lower and upper limits in the lemma are independent of the representation of $x$. Let $x=\sum_{i=1}^{\infty} \varepsilon_{i}^{\prime} \rho^{i}$ be another representation and let $x_{n}^{\prime}=\sum_{i=1}^{n} \varepsilon_{i}^{\prime} \rho^{i}$. Since both $x_{n}$ and $x_{n}^{\prime}$ are contained in $I\left(x,(1+\ell) \rho^{n}\right)$, by condition A, there are at most $2 C(1+\ell)$ distinct values of $S_{n}$ between $x_{n}$ and $x_{n}^{\prime}$, where $C$ is the constant in condition A. By applying condition B to these distinct values successively on every two consecutive ones, we obtain

$$
1 /\left(\theta_{n}\right)^{2 C(1+\ell)} \leq \#\left\langle x_{n}^{\prime}\right\rangle / \#\left\langle x_{n}\right\rangle \leq\left(\theta_{n}\right)^{2 C(1+\ell)} .
$$

By using $\lim _{n \rightarrow \infty}\left(\log \theta_{n} / n\right)=0$, we see that both $\log \#\left\langle x_{n}^{\prime}\right\rangle / n$ and $\log \#\left\langle x_{n}\right\rangle / n$ have the same lower and upper limits.

We only prove the first formula; the proof for the second one is the same. Let $v \in I\left(x,(1+\ell) \rho^{n}\right) \cap V_{n}$ be such that $\#\langle v\rangle=\max \left\{\#\langle\xi\rangle: \xi \in I\left(x,(1+\ell) \rho^{n}\right) \cap V_{n}\right\}$. By condition A, we have

$$
\mu_{n}\left(I\left(x,(1+\ell) \rho^{n}\right)\right) \leq 2 C(1+\ell) 2^{-n} \#\langle v\rangle .
$$

By using Lemma 2.2 and noting that both $\log \#\langle v\rangle / n$ and $\log \#\left\langle x_{n}\right\rangle / n$ have the same lower and upper limits, we deduce that

$$
\begin{aligned}
\bar{d}(\mu, x) & \geq \varlimsup_{n \rightarrow \infty} \frac{\log \left(2 C(1+\ell) 2^{-n} \#\langle v\rangle\right)}{n \log \rho} \\
& =\varlimsup_{n \rightarrow \infty} \frac{\log \#\left\langle x_{n}\right\rangle}{n \log \rho}-\frac{\log 2}{\log \rho} .
\end{aligned}
$$

The reverse inequality can be proved by applying (2.3) and a similar argument.

Corollary 2.7. Let $1 / 2<\rho<1$. Suppose that $S$ satisfies both condition A and condition B. Assume that a series representation $x=\sum_{i=1}^{\infty} \varepsilon_{i} \rho^{i}$ and a sequence of positive integers $n_{1}<n_{2}<\cdots$ exist such that

(i) $\lim _{k \rightarrow \infty} n_{k} / n_{k-1}=1$; and

(ii) $\lim _{k \rightarrow \infty}\left(\log \#\left\langle x_{n_{k}}\right\rangle / n_{k}\right)=\alpha$ exists.

Then $d(\mu, x)$ exists and $d(\mu, x)=(\alpha-\log 2) / \log \rho$.

Proof. By Lemma 2.6, it suffices to show that the assumptions imply

$$
\lim _{n \rightarrow \infty}\left(\log \#\left\langle x_{n}\right\rangle / n\right)=\alpha .
$$

For any integer $n$, let $n_{k-1} \leq n<n_{k}$. Since $\#\left\langle x_{n}\right\rangle$ is increasing, we have

$$
\frac{\log \#\left\langle x_{n}\right\rangle}{n} \leq \frac{\log \#\left\langle x_{n_{k}}\right\rangle}{n_{k-1}}=\frac{\left[\log \#\left\langle x_{n_{k}}\right\rangle\right] / n_{k}}{n_{k-1} / n_{k}}
$$

and

$$
\frac{\log \#\left\langle x_{n}\right\rangle}{n} \geq \frac{\left[\log \#\left\langle x_{n_{k-1}}\right\rangle\right] / n_{k-1}}{n_{k} / n_{k-1}} .
$$

Passing to the limit and using (i) and (ii), we obtain $\lim _{n \rightarrow \infty}\left(\log \#\left\langle x_{n}\right\rangle / n\right)=\alpha$. 
We will show next that if $\rho$ is a root of (1.1), then $S$ satisfies both condition A and condition B. Thus all lemmas in this section will apply.

As before, $x_{n}(1)<x_{n}(2)<\cdots<x_{n}\left(i_{n}\right)$ will denote all distinct values of $S_{n}$. Let $\delta\left(S_{n}\right)=\min \left\{x_{n}(j)-x_{n}(j-1): 2 \leq j \leq i_{n}\right\}$.

Lemma 2.8. Let $1 / 2<\rho<1$. If $\delta\left(S_{n}\right) \geq 2 \rho^{n+1}$ for all $n$, then $S$ satisfies both condition $\mathrm{A}$ and condition $\mathrm{B}$.

Proof. Clearly the assumption implies that $S$ satisfies condition A. To show that $S$ also satisfies condition $\mathrm{B}$, we will show by induction that

$$
1 / n \leq \#\left\langle x_{n}(j+1)\right\rangle / \#\left\langle x_{n}(j)\right\rangle \leq n, \quad \text { for all } n \text { and for all } 1 \leq j<i_{n} .
$$

For $n=1,-\rho$ and $\rho$ are the only two values of $S_{1}$, so (2.6) is clearly true. Suppose that (2.6) is true for $n=k$. Let $x_{k+1}(i)$ be any value of $S_{k+1}$ (without loss of generality, we may assume that it is not one of the two extreme values). Then $x_{k+1}(i) \in\left[x_{k}(j), x_{k}(j+1)\right)$ for some $1 \leq j<i_{k}$. The assumption $\delta\left(S_{k}\right) \geq 2 \rho^{k+1}$ implies that either $x_{k+1}(i)=x_{k}(j+1)-\rho^{k+1}$ or $x_{k+1}(i)=x_{k}(j)+\rho^{k+1}$. Since for either case the proof is the same, we assume that $x_{k+1}(i)=x_{k}(j+1)-\rho^{k+1}$. Obviously if $x_{k}(j+1)-x_{k}(j)=2 \rho^{k+1}$, then $x_{k+1}(i)$ also equals $x_{k}(j)+\rho^{k+1}$. In any case we have $\#\left\langle x_{k+1}(i)\right\rangle \leq \#\left\langle x_{k}(j)\right\rangle+\#\left\langle x_{k}(j+1)\right\rangle$ and $x_{k+1}(i+1)=x_{k}(j+1)+\rho^{k+1}$ $\left(\right.$ also $x_{k+1}(i+1)=x_{k}(j+2)-\rho^{k+1}$ if $\left.x_{k}(j+2)-x_{k}(j+1)=2 \rho^{k+1}\right)$. By the induction hypothesis for $n=k$ we get

$$
\frac{\#\left\langle x_{k+1}(i)\right\rangle}{\#\left\langle x_{k+1}(i+1)\right\rangle} \leq \frac{\#\left\langle x_{k}(j)\right\rangle+\#\left\langle x_{k}(j+1)\right\rangle}{\#\left\langle x_{k}(j+1)\right\rangle} \leq k+1
$$

and

$$
\frac{\#\left\langle x_{k+1}(i)\right\rangle}{\#\left\langle x_{k+1}(i+1)\right\rangle} \geq \frac{\#\left\langle x_{k}(j+1)\right\rangle}{\#\left\langle x_{k}(j+1)\right\rangle+\#\left\langle x_{k}(j+2)\right\rangle} \geq \frac{1}{k+1} .
$$

Hence (2.6) is true for $n=k+1$.

Proposition 2.9. If $\rho$ is a root of (1.1), then $S$ satisfies both condition A and condition $\mathrm{B}$.

Proof. We will prove the following claim.

Claim. For any $n$ and for any $1 \leq j<i_{n}$, there is some $0 \leq m \leq s-1$ such that $x_{n}(j+1)-x_{n}(j)=2 \rho^{n-m}\left(1-\rho-\rho^{2}-\cdots-\rho^{m}\right)$.

Since $1-\rho-\rho^{2}-\cdots-\rho^{s}=0$, the claim implies that

$$
x_{n}(j+1)-x_{n}(j)=2 \rho^{n-m}\left(\rho^{m+1}+\rho^{m+2}+\cdots+\rho^{s}\right) \geq 2 \rho^{n+1}
$$

Thus the proposition follows from this and Lemma 2.8. The claim can be proved by induction. For $n=1, S_{1}$ has only two values $\rho$ and $-\rho$. Hence the claim is true with $m=0$. Suppose that it is true for $n=k$. Let $x_{k+1}(i)$ be any value of $S_{k+1}$. As before, assume that $x_{k+1}(i) \in\left[x_{k}(j), x_{k}(j+1)\right)$ for some $j$. By the induction assumption and by $(2.7)$ we have $\delta\left(S_{k}\right) \geq 2 \rho^{k+1}$. Thus we can further assume that $x_{k+1}(i)=x_{k}(j)+\rho^{k+1}$. This implies that

$$
x_{k+1}(i+1)= \begin{cases}x_{k}(j+1)-\rho^{k+1}, & \text { if } x_{k}(j+1)-x_{k}(j)>2 \rho^{k+1}, \\ x_{k}(j+1)+\rho^{k+1}, & \text { if } x_{k}(j+1)-x_{k}(j)=2 \rho^{k+1}\end{cases}
$$


Accordingly

$$
\begin{aligned}
x_{k+1}(i+1)-x_{k+1}(i) \\
\quad=\left\{\begin{array}{l}
x_{k}(j+1)-x_{k}(j)-2 \rho^{k+1}, \quad \text { if } x_{k}(j+1)-x_{k}(j)>2 \rho^{k+1}, \\
2 \rho^{k+1}, \quad \text { if } x_{k}(j+1)-x_{k}(j)=2 \rho^{k+1} .
\end{array}\right.
\end{aligned}
$$

From (2.7) we see that $x_{k}(j+1)-x_{k}(j)=2 \rho^{k+1}$ if and only if $m=s-1$ (or equivalently, $x_{k}(j+1)-x_{k}(j)=2 \rho^{k-m}\left(1-\rho-\cdots-\rho^{m}\right)>2 \rho^{k+1}$ if and only if $0 \leq m \leq s-2)$. So there is some $0 \leq m \leq s-2$ such that the first case of (2.8) equals

$$
\begin{aligned}
x_{k}(j+1)-x_{k}(j)-2 \rho^{k+1} & =2 \rho^{k-m}\left[\left(1-\rho-\cdots-\rho^{m}\right)-\rho^{m+1}\right] \\
& =2 \rho^{(k+1)-(m+1)}\left(1-\rho-\cdots-\rho^{m+1}\right) \\
& =2 \rho^{(k+1)-q}\left(1-\rho-\cdots-\rho^{q}\right),
\end{aligned}
$$

where $q=m+1$. Hence $1 \leq q \leq s-1$. The second case of (2.8) is obtained by setting $q=0$ in (2.9). So the claim is true.

\section{THE ZERO ELEMENTS}

For $1 / 2<\rho<1$, let $\mathbb{Z}_{n}$ be the set of zero elements as defined in $\S 1$. Clearly $\mathbb{Z}_{n} \neq \varnothing$ for every $n$ since it contains the zero element for which $a_{i}=0$ for all $i$, which is called the unit zero. For any fixed $\mathbf{b}=\left(b_{1}, \ldots, b_{n}\right) \in\{-1,0,1\}^{n}$, we say that $\mathbf{b}$ contains a zero element $\mathbf{a}=\left(a_{1}, \ldots, a_{n}\right)$ (or $\mathbf{a}$ is a zero element of $\mathbf{b}$ ) if

$$
a_{i}=b_{i}, \quad \text { for all } i \text { for which } a_{i} \neq 0 .
$$

We agree that any such $\mathbf{b}$ contains the unit zero. For example, if $\rho$ satisfies $1-$ $\rho-\rho^{2}=0$ and if $\mathbf{b}=(1,-1,-1,1,1)$, then $\mathbf{b}$ contains the following three zero elements: The unit zero, $(1,-1,-1,0,0)$ and $(0,0,-1,1,1)$. We use $\mathbb{Z}_{n}(\mathbf{b})$ and $\# \mathbb{Z}_{n}(\mathbf{b})$ to denote the set consisting of all zero elements of $\mathbf{b}$ and its cardinality, respectively. A zero element $\mathbf{a} \in \mathbb{Z}_{n}$ is called an atom if $\mathbf{a}$ contains no zero element other than the unit zero and itself.

Lemma 3.1. Let $1 / 2<\rho<1$ and let $x \in[-\ell, \ell]$. Then for any fixed series representation $x=\sum_{i=1}^{\infty} \varepsilon_{i} \rho^{i}$, for any $n$ and for any $\boldsymbol{\tau}^{(\mathbf{n})} \in\left\langle x_{n}\right\rangle$, we have $\#\left\langle x_{n}\right\rangle=$ $\# \mathbb{Z}_{n}\left(\boldsymbol{\tau}^{(\mathbf{n})}\right)$. In particular, every member in $\left\langle x_{n}\right\rangle$ contains the same number of zero elements.

Proof. Let $\boldsymbol{\tau}^{(\mathbf{n})}=\left(\tau_{1}, \ldots, \tau_{n}\right)$, where $\tau_{i}= \pm 1$, be an arbitrary fixed member in $\left\langle x_{n}\right\rangle$. Define a mapping $f=f_{\boldsymbol{\tau}^{(\mathbf{n})}}:\left\langle x_{n}\right\rangle \rightarrow \mathbb{Z}_{n}\left(\boldsymbol{\tau}^{(\mathbf{n})}\right)$ by the following process: For any $\boldsymbol{\omega}^{(\mathbf{n})}=\left(\omega_{1}, \ldots, \omega_{n}\right) \in\left\langle x_{n}\right\rangle$, where $\omega_{i}= \pm 1$, we have

$$
0=\sum_{i=1}^{n} \tau_{i} \rho^{i}-\sum_{i=1}^{n} \omega_{i} \rho^{i}=2 \sum_{i=1}^{n} a_{i} \rho^{i}
$$

where

$$
a_{i}=\left(\tau_{i}-\omega_{i}\right) / 2= \begin{cases}0, & \text { if } \tau_{i}=\omega_{i}, \\ 1, & \text { if } \tau_{i} \neq \omega_{i} \text { and } \tau_{i}=1 \\ -1, & \text { if } \tau_{i} \neq \omega_{i} \text { and } \tau_{i}=-1\end{cases}
$$


It follows that $a_{i}=\tau_{i}$ whenever $a_{i} \neq 0$. By $(3.1),\left(a_{1}, \ldots, a_{n}\right)$ is a zero element of $\boldsymbol{\tau}^{(\mathbf{n})}$. Define $f\left(\boldsymbol{\omega}^{(\mathbf{n})}\right)=\left(a_{1}, \ldots, a_{n}\right)$. By using (3.2), a routine check reveals that $f$ is one-to-one and hence the lemma follows.

Two zero elements $\mathbf{a}=\left(a_{1}, \ldots, a_{n}\right)$ and $\mathbf{b}=\left(b_{1}, \ldots, b_{n}\right)$ in $\mathbb{Z}_{n}$ is said to be mutually disjoint if $b_{i}=0$ whenever $a_{i} \neq 0$ (or equivalently $a_{i}=0$ whenever $\left.b_{i} \neq 0\right)$ for $i=1, \ldots, n$. If $\mathbf{a}$ and $\mathbf{b}$ are mutually disjoint, we see that $\mathbf{c}=\left(a_{1}+\right.$ $\left.b_{1}, \ldots, a_{n}+b_{n}\right)$ is also a zero element, and $\mathbf{c}$ is called a direct sum of $\mathbf{a}$ and $\mathbf{b}$, denoted by $\mathbf{c}=\mathbf{a} \oplus \mathbf{b}$. Clearly $\mathbf{a} \oplus \mathbf{b}=\mathbf{b} \oplus \mathbf{a}$.

Theorem 3.2. Let $1 / 2<\rho<1$, then $\mathbf{a}$ is a zero element in $\mathbb{Z}_{n}$ if and only if $\mathbf{a}=\mathbf{a}_{\mathbf{1}} \oplus \mathbf{a}_{\mathbf{2}} \oplus \cdots \oplus \mathbf{a}_{\mathbf{k}}$ for some pairwise mutually disjoint atoms $\mathbf{a}_{\mathbf{1}}, \mathbf{a}_{\mathbf{2}}, \ldots, \mathbf{a}_{\mathbf{k}}$, where $k \geq 1$.

Proof. The proof for the "if" part is trivial, so we show only the "only if" part.

Let $\mathbf{a}=\left(a_{1}, \ldots, a_{n}\right)$ be a zero element. Without loss of generality, assume that $\mathbf{a}$ contains a nontrivial zero element $\mathbf{a}_{\mathbf{1}}$. We can actually assume that $\mathbf{a}_{\mathbf{1}}$ is already an atom, for otherwise we may apply the above argument to $\mathbf{a}_{\mathbf{1}}$ until an atom is obtained. Let $\mathbf{a}_{\mathbf{1}}=\left(a_{1}^{\prime}, \ldots, a_{n}^{\prime}\right)$ and let $\mathbf{b}=\left(a_{1}-a_{1}^{\prime}, \ldots, a_{n}-a_{n}^{\prime}\right)$. Since $\mathbf{a}_{\mathbf{1}}$ is a zero element of $\mathbf{a}$, by (3.1) we can conclude that $a_{i}-a_{i}^{\prime}=0$ whenever $a_{i}^{\prime} \neq 0$. So $\mathbf{a}_{\mathbf{1}}$ and $\mathbf{b}$ are mutually disjoint zero elements and $\mathbf{a}=\mathbf{a}_{\mathbf{1}} \oplus \mathbf{b}$. If $\mathbf{b}$ is an atom, the theorem is proved. Otherwise, the above decomposition can be applied to $\mathbf{b}$ and its decomposed elements a finite number of times until a complete atom representation of $\mathbf{a}$ is obtained.

For any $\varepsilon=\left(\varepsilon_{1}, \varepsilon_{2}, \ldots\right) \in\{-1,1\}^{\infty}$, let $\boldsymbol{\varepsilon}^{(\mathbf{n})}=\left(\varepsilon_{1}, \ldots, \varepsilon_{n}\right)$ and let

$$
p_{n}=p_{n}(\varepsilon)=\# \mathbb{Z}_{n}\left(\varepsilon^{(\mathbf{n})}\right) \text {. }
$$

If $x=\sum_{i=1}^{\infty} \varepsilon_{i} \rho^{i}$, then the components of $\varepsilon^{(\mathbf{n})}$ are the coefficients of $x_{n}$. By Lemma 3.1, for all $n$, we have

$$
p_{n}=\#\left\langle x_{n}\right\rangle \text {. }
$$

Remark 3.3. It is easy to see that $\left(a_{1}, \ldots, a_{n-1}\right)$ is a zero element of $\boldsymbol{\varepsilon}^{(\mathbf{n}-\mathbf{1})}$ if and only if $\left(a_{1}, \ldots, a_{n-1}, 0\right)$ is a zero element of $\boldsymbol{\varepsilon}^{(\mathbf{n})}$. Hence $p_{n} \geq p_{n-1}$ for all $n>1$. This in turn explains (2.4).

Lemma 3.4. Let $1 / 2<\rho<1$, and let $\boldsymbol{\varepsilon}^{(\mathbf{n})}$ and $p_{n}$ be defined as above. Then $p_{n}>p_{n-1}$ if and only if there is an atom $\mathbf{a}=\left(a_{1}, \ldots, a_{n}\right)$ of $\varepsilon^{(\mathbf{n})}$ such that $a_{n}=\varepsilon_{n} \neq 0$.

Proof. Assume that $p_{n}>p_{n-1}$. Since $\boldsymbol{\varepsilon}^{(\mathbf{n})}$ contains more zero elements that $\boldsymbol{\varepsilon}^{(\mathbf{n}-\mathbf{1})}$ does, $\boldsymbol{\varepsilon}^{(\mathbf{n})}$ contains a zero element $\mathbf{a}=\left(a_{1}, \ldots, a_{n}\right)$ for which $a_{n} \neq 0$. Thus $a_{n}=\varepsilon_{n}$ by (3.1). Clearly a can be assumed to be an atom by Theorem 3.2. Conversely, suppose there is an atom $\mathbf{a}=\left(a_{1}, \ldots, a_{n}\right)$ of $\boldsymbol{\varepsilon}^{(\mathbf{n})}$ such that $a_{n}=\varepsilon_{n} \neq 0$. By Remark 3.3, the zero elements of $\varepsilon^{(\mathbf{n})}$ will include at least all zero elements of $\varepsilon^{(\mathbf{n}-\mathbf{1})}$ together with a, so $p_{n}>p_{n-1}$.

For any $\mathbf{c}=\left(c_{1}, \ldots, c_{n}\right) \in\{-1,0,1\}^{n}$ for which $c_{i} \neq 0$ for at least one index $i$, define

$$
m(\mathbf{c})=\min \left\{i: c_{i} \neq 0\right\}, \quad \text { and } \quad M(\mathbf{c})=\max \left\{i: c_{i} \neq 0\right\}
$$


For $\mathbf{a}, \mathbf{b} \in \mathbb{Z}_{\mathbf{n}}$, we say that $\mathbf{a}$ and $\mathbf{b}$ are strongly mutually disjoint if either $m(\mathbf{a})>$ $M(\mathbf{b})$ or $m(\mathbf{b})>M(\mathbf{a})$. It is obvious that strongly mutually disjoint implies mutually disjoint.

If $\mathbf{a}=\left(a_{1}, \ldots, a_{n}\right)$ is a zero element of $\mathbf{c}=\left(c_{1}, \ldots, c_{n}\right)$, let $\mathbf{c} \ominus \mathbf{a}=\left(c_{1}-\right.$ $\left.a_{1}, \ldots, c_{n}-a_{n}\right)$. Let $\varepsilon^{(\mathbf{n})}$ and $\mathbf{a}$ be defined as in Lemma 3.4. If $p_{n}>p_{n-1}$, then the final entry of $\varepsilon^{(\mathbf{n})} \ominus \mathbf{a}$ equals $\varepsilon_{n}-a_{n}=0$. With regard to the computation of zero elements, $\boldsymbol{\varepsilon}^{(\mathbf{n})} \ominus \mathbf{a}$ can be identified as $\left(\varepsilon_{1}-a_{1}, \ldots, \varepsilon_{n-1}-a_{n-1}\right)$, for by Remark 3.3 they contain the same number of zero elements.

Lemma 3.5. Let $\rho, \varepsilon^{(\mathbf{n})}$ and $p_{n}$ be defined as in Lemma 3.4 and suppose that $p_{n}>$ $p_{n-1}$. Then

$$
p_{n}=p_{n-1}+\sum_{i=1}^{k} \# \mathbb{Z}_{n-1}\left(\varepsilon^{(\mathbf{n})} \ominus \mathbf{a}_{\mathbf{i}}\right),
$$

where $\mathbf{a}_{\mathbf{i}}$, for $i=1, \ldots, k$, are all atoms of $\varepsilon^{(\mathbf{n})}$ whose final entries equal $\varepsilon_{n} \neq 0$. If we further assume that every zero element of $\varepsilon^{(\mathbf{n})}$ is a direct sum of some pairwise strongly mutually disjoint atoms, then $p_{n}=p_{n-1}+\sum_{i=1}^{k} p_{m\left(\mathbf{a}_{\mathbf{i}}\right)-1}$.

Proof. Let $\mathbf{a}=\left(a_{1}, \ldots, a_{n}\right)$ be an arbitrary zero element of $\boldsymbol{\varepsilon}^{(\mathbf{n})}$. If $a_{n}=0$, then a can be identified as a zero element of $\varepsilon^{(\mathbf{n}-\mathbf{1})}$. If $a_{n} \neq 0$, then $a_{n}=\varepsilon_{n}$ by (3.1). Theorem 3.2 and Lemma 3.4 imply that $\mathbf{a}=\mathbf{b} \oplus \mathbf{a}_{\mathbf{i}}$ for some zero element $\mathbf{b}$ of $\boldsymbol{\varepsilon}^{(\mathbf{n})}$ and some atom $\mathbf{a}_{\mathbf{i}}$ whose final entry equals $\varepsilon_{n}$. Obviously $\mathbf{b}$ is a zero element contained in $\varepsilon^{(\mathbf{n})} \ominus \mathbf{a}_{\mathbf{i}}$, since $\mathbf{b}$ and $\mathbf{a}_{\mathbf{i}}$ are mutually disjoint. We thus have proved that any zero element of $\varepsilon^{(\mathbf{n})}$ is either a zero element of $\varepsilon^{(\mathbf{n}-\mathbf{1})}$ or a direct sum of $\mathbf{a}_{\mathbf{i}}$ and a zero element contained in $\boldsymbol{\varepsilon}^{(\mathbf{n})} \ominus \mathbf{a}_{\mathbf{i}}$. Hence

$$
p_{n}=p_{n-1}+\sum_{i=1}^{k} \# \mathbb{Z}_{n-1}\left(\varepsilon^{(\mathbf{n})} \ominus \mathbf{a}_{\mathbf{i}}\right) .
$$

If we further assume that every zero element of $\varepsilon^{(\mathbf{n})}$ is a direct sum of some pairwise strongly mutually disjoint atoms (this direct sum must be unique, see the proof of Theorem 3.7), then $M(\mathbf{b})<m\left(\mathbf{a}_{\mathbf{i}}\right)$, so $\mathbf{b}$ is a zero element contained in $\left(\varepsilon_{1}, \ldots, \varepsilon_{m\left(\mathbf{a}_{\mathbf{i}}\right)-1}, 0, \ldots, 0\right)$. Therefore $p_{n}=p_{n-1}+\sum_{i=1}^{k} p_{m\left(\mathbf{a}_{\mathbf{i}}\right)-1}$.

We will study the forms of zero elements for the case $\rho=(\sqrt{5}-1) / 2$, i.e., $\rho$ satisfies the equation $1-\rho-\rho^{2}=0$. For the general case in which $\rho$ satisfying (1.1), we will indicate the changes needed for these forms. Due to the fact that $\rho^{n}=\rho^{n+1}+\rho^{n+2}$, we see that $0=1-\rho-\rho^{2}=1-\rho-\rho^{3}-\rho^{4}=1-\rho-\rho^{3}-\rho^{5}-\rho^{6}=$ $\cdots$. Therefore $\pm(0, \ldots, 0,1,-1,-1,0, \ldots, 0), \pm(0, \ldots, 0,1,-1,0,-1,-1,0, \ldots, 0)$, $\pm(0, \ldots, 0,1,-1,0,-1,0,-1,-1,0, \ldots, 0), \ldots$, are zero elements in $\mathbb{Z}_{n}$ and contain no nontrivial zero element, so all of them are atoms. We will show that all atoms of $\mathbb{Z}_{n}$ are precisely of these forms. Let $\mathbb{A}_{n}$ be the subset consisting of all $n$-tuples with the forms as above. More precisely, let

$$
\begin{aligned}
\mathbb{A}_{n}=\{\mathbf{a}= & \pm\left(a_{1}, \ldots, a_{n}\right): M(\mathbf{a})-m(\mathbf{a}) \text { is even, } a_{m(\mathbf{a})}=1, a_{i}=-1 \text { for } i=m(\mathbf{a})+1 \\
& \left.m(\mathbf{a})+3, \ldots, M(\mathbf{a})-3, M(\mathbf{a})-1, M(\mathbf{a}), \text { and } a_{i}=0 \text { for all other } i\right\}
\end{aligned}
$$

Lemma 3.6. Let $\rho=(\sqrt{5}-1) / 2$. For any $\mathbf{a} \in \mathbb{Z}_{n}$, either $\mathbf{a} \in \mathbb{A}_{n}$ or $\mathbf{a}=\mathbf{a}_{\mathbf{1}} \oplus \mathbf{a}_{2}$ for some strongly mutually disjoint $\mathbf{a}_{\mathbf{1}} \in \mathbb{A}_{n}$ and $\mathbf{a}_{\mathbf{2}} \in \mathbb{Z}_{n}$. 
Proof. Let $\mathbf{a}=\left(a_{1}, \ldots, a_{n}\right)$. Without loss of generality, assume that $m(\mathbf{a})=1$ and $a_{1}=1$. Then

$$
\sum_{i=1}^{n} a_{i} \rho^{i}=\rho+\sum_{i=2}^{n} a_{i} \rho^{i}=0 .
$$

Using $1-\rho=\rho^{2}$, for any $k \geq 1$ we have

$$
\sum_{i=k}^{\infty} \rho^{i}=\rho^{k} /(1-\rho)=\rho^{k-2} .
$$

It follows that

$$
0>\rho+a_{2} \rho^{2}-\sum_{i=3}^{\infty} \rho^{i}=\rho+a_{2} \rho^{2}-\rho=a_{2} \rho^{2} .
$$

Hence $a_{2}=-1$. Using (3.4) and (3.5) again, we have

$$
0=\rho-\rho^{2}+\sum_{i=3}^{n} a_{i} \rho^{i}=\rho^{3}+a_{3} \rho^{3}+\sum_{i=4}^{n} a_{i} \rho^{i}>\left(1+a_{3}\right) \rho^{3}-\rho^{2} .
$$

Clearly $a_{3} \neq 1$, otherwise the right-hand side above would be strictly greater than zero. Therefore either $a_{3}=-1$ or $a_{3}=0$. First assume that $a_{3}=-1$. If $M(\mathbf{a})=3$, then $\mathbf{a}=(1,-1,-1,0, \ldots, 0) \in \mathbb{A}_{n} ;$ if $M(\mathbf{a})>3$, then we can write $\mathbf{a}=\mathbf{a}_{\mathbf{1}} \oplus \mathbf{a}_{\mathbf{2}}$ with $\mathbf{a}_{1}=(1,-1,-1,0, \ldots, 0) \in \mathbb{A}_{n}$ and $\mathbf{a}_{2}=\left(0,0,0, a_{4}, \ldots, a_{n}\right) \in \mathbb{Z}_{n}$. This proves the lemma. Next, if $a_{3}=0$, then

$$
0=\rho^{3}+\sum_{i=4}^{n} a_{i} \rho^{i}=\rho^{2}\left(\rho+\sum_{i=2}^{n-2} a_{i+2} \rho^{i}\right) .
$$

The expression inside the parentheses in (3.6) is of the form (3.4) and equals zero. Applying the above argument we conclude that $a_{4}=-1$ and that either $a_{5}=-1$ or $a_{5}=0$. Suppose that $a_{5}=-1$. Then $\mathbf{a} \in \mathbb{A}_{n}$ if $M(\mathbf{a})=5$; if $M(\mathbf{a})>5$, then $\mathbf{a}=$ $\mathbf{a}_{\mathbf{1}} \oplus \mathbf{a}_{\mathbf{2}}$ with $\mathbf{a}_{\mathbf{1}}=(1,-1,0,-1,-1,0, \ldots, 0) \in \mathbb{A}_{n}$ and $\mathbf{a}_{\mathbf{2}}=\left(0, \ldots, 0, a_{6}, \ldots, a_{n}\right) \in$ $\mathbb{Z}_{n}$, proving the lemma. If $a_{5}=0$, then

$$
0=\rho^{3}-\rho^{4}+\sum_{i=6}^{n} a_{i} \rho^{i}=\rho^{4}\left(\rho+\sum_{i=2}^{n-4} a_{i+4} \rho^{i}\right) .
$$

Repeating the same argument a finite number of times we obtain the required decomposition.

Theorem 3.7. Let $\rho=(\sqrt{5}-1) / 2$. $\mathbb{A}_{n}$ is precisely the set of all atoms of $\mathbb{Z}_{n}$. For any $\mathbf{a} \in \mathbb{Z}_{n}$, then $\mathbf{a}=\mathbf{a}_{\mathbf{1}} \oplus \mathbf{a}_{\mathbf{2}} \oplus \cdots \oplus \mathbf{a}_{\mathbf{k}}$ for some pairwise strongly mutually disjoint atoms $\mathbf{a}_{\mathbf{1}}, \mathbf{a}_{\mathbf{1}}, \ldots, \mathbf{a}_{\mathbf{k}}$, where $k \geq 1$. Furthermore, this decomposition is unique.

Proof. It is easy to verify that members of $\mathbb{A}_{n}$ are atoms. Conversely, let $\mathbf{a} \in \mathbb{Z}_{n}$ be any atom. Since it contains no nontrivial zero element, Lemma 3.6 implies that $\mathbf{a} \in \mathbb{A}_{n}$, proving the first statement. Obviously the atom decomposition part is immediate from Lemma 3.6.

It remains to show the uniqueness. Let $\mathbf{a}=\left(a_{1}, \ldots, a_{n}\right)$. Suppose that $\mathbf{a}=$ $\mathbf{b}_{\mathbf{1}} \oplus \mathbf{b}_{\mathbf{2}} \oplus \cdots \oplus \mathbf{b}_{\mathbf{j}}$ is another such decomposition and assume that both $\mathbf{a}_{\mathbf{1}}$ and $\mathbf{b}_{\mathbf{1}}$ contain the entry $a_{1}$. Write $\mathbf{a}^{\prime}=\mathbf{a}_{\mathbf{2}} \oplus \cdots \oplus \mathbf{a}_{\mathbf{k}}$ and $\mathbf{b}^{\prime}=\mathbf{b}_{\mathbf{2}} \oplus \cdots \oplus \mathbf{b}_{\mathbf{j}}$. If $\mathbf{a}_{\mathbf{1}} \neq \mathbf{b}_{\mathbf{1}}$, without loss of generality, assume that $M\left(\mathbf{a}_{\mathbf{1}}\right) \leq M\left(\mathbf{b}_{\mathbf{1}}\right)$. Since $\mathbf{a}_{\mathbf{1}}$ is not a zero element of $\mathbf{b}_{\mathbf{1}}$ (both of them are atoms), hence there is an entry $a_{i}^{\prime}$ of $\mathbf{a}_{\mathbf{1}}$ and an 
entry $b_{i}^{\prime}$ of $\mathbf{b}_{\mathbf{1}}$ such that $a_{i}^{\prime} \neq 0$ and $a_{i}^{\prime} \neq b_{i}^{\prime}$ (so $i \leq M\left(\mathbf{a}_{\mathbf{1}}\right)$ ). This forces $b_{i}^{\prime}=0$, otherwise by (3.1) we will have $b_{i}^{\prime}=a_{i}=a_{i}^{\prime}$. This implies that $i<M\left(\mathbf{b}_{\mathbf{1}}\right)$. Note that $\mathbf{a}=\mathbf{b}_{\mathbf{1}} \oplus \mathbf{b}^{\prime}$, so there must be an entry $b_{i}$ of $\mathbf{b}^{\prime}$ such that $b_{i}=a_{i}(\neq 0)$. So $m\left(\mathbf{b}^{\prime}\right) \leq i<M\left(\mathbf{b}_{\mathbf{1}}\right)$, contradicting the fact that $\mathbf{b}_{\mathbf{1}}$ and $\mathbf{b}^{\prime}$ are strongly mutually disjoint. Therefore $\mathbf{a}_{\mathbf{1}}=\mathbf{b}_{\mathbf{1}}$. It follows that $\mathbf{a}^{\prime}=\mathbf{a} \ominus \mathbf{a}_{\mathbf{1}}=\mathbf{a} \ominus \mathbf{b}_{\mathbf{1}}=\mathbf{b}^{\prime}$. Applying the same argument to $\mathbf{a}^{\prime}=\mathbf{b}^{\prime}$ a finite number of times, we obtain $k=j$ and $\mathbf{a}_{\mathbf{i}}=\mathbf{b}_{\mathbf{i}}$ for $i=1, \ldots, k$.

Remark 3.8. If $\rho$ is a root of $(1.1)$, then $\rho^{j s}=\rho^{j s} \sum_{i=1}^{s} \rho^{i}$, for $j=0,1, \ldots$ Therefore

$$
0=1-\sum_{i=1}^{s} \rho^{i}=1-\sum_{i=1}^{s-1} \rho^{i}-\sum_{i=s+1}^{2 s} \rho^{i}=1-\sum_{i=1}^{s-1} \rho^{i}-\sum_{i=s+1}^{2 s-1} \rho^{i}-\sum_{i=2 s+1}^{3 s} \rho^{i}=\cdots .
$$

From this it can be shown by a similar argument that the subset

$$
\begin{aligned}
& \mathbb{A}_{n}^{\prime}=\left\{\mathbf{a}= \pm\left(a_{1}, \ldots, a_{n}\right): M(\mathbf{a})-m(\mathbf{a}) \text { is a multiple of } s\right. \\
& \quad a_{m(\mathbf{a})=1, a_{i}=-1, \text { for } i=M(\mathbf{a}) \text { and }} \\
& i=m(\mathbf{a})+k s+j, \text { where } k=0,1, \ldots,(M(\mathbf{a})-m(\mathbf{a})) / s-1 \text { and } \\
& \left.\quad j=1,2, \ldots, s-1, \text { and } a_{i}=0 \text { for all other } i\right\}
\end{aligned}
$$

will precisely consist of all atoms of $\mathbb{Z}_{n}$. Similarly we have Theorem 3.7 , the unique decomposition of a zero element as pairwise strongly mutually disjoint atoms.

Atoms in $\mathbb{A}_{n}$ have the following two properties which will be used later.

Proposition 3.9. Let $\rho=(\sqrt{5}-1) / 2$. Let $\mathbf{a}=\left(a_{1}, \ldots, a_{n}\right) \in \mathbb{A}_{n}$. Suppose $a_{i}=0$, for some $i$ satisfying $m(\mathbf{a})<i<M(\mathbf{a})$. Then $i-m(\mathbf{a})$ is an even integer.

Proof. The proposition can be easily verified from the definition of $\mathbb{A}_{n}$.

Proposition 3.10. Let $\rho=(\sqrt{5}-1) / 2$. Let $\mathbf{a}$ and $\mathbf{b}$ be two atoms of $\boldsymbol{\varepsilon}^{(\mathbf{n})}=$ $\left(\varepsilon_{1}, \ldots, \varepsilon_{n}\right)$ that are not strongly mutually disjoint. Then both $M(\mathbf{a})-M(\mathbf{b})$ and $m(\mathbf{a})-m(\mathbf{b})$ must be even (or zero).

Proof. By the definition of $\mathbb{A}_{\mathbf{n}}$, it suffices to show that $m(\mathbf{a})-m(\mathbf{b})$ is even (or zero). Write $\mathbf{a}=\left(a_{1}, \ldots, a_{n}\right)$ and $\mathbf{b}=\left(b_{1}, \ldots, b_{n}\right)$. Since $\mathbf{a}$ and $\mathbf{b}$ are not strongly mutually disjoint, without loss of generality, assume that $a_{m(\mathbf{a})}=1$ and that $m(\mathbf{a})<$ $m(\mathbf{b})<M(\mathbf{a})$ (note that the case $m(\mathbf{b})=m(\mathbf{a})$ or $m(\mathbf{b})=M(\mathbf{a})$ already implies that $m(\mathbf{a})-m(\mathbf{b})$ is even or zero). By (3.1) and by the structure of atoms we obtain

(3.7) $\varepsilon_{i}=a_{i}=-1, \quad$ for $i=m(\mathbf{a})+1, m(\mathbf{a})+3, \ldots, M(\mathbf{a})-3, M(\mathbf{a})-1, M(\mathbf{a})$.

Claim. $a_{m(\mathbf{b})} \neq 1$.

Actually if $a_{m(\mathbf{b})}=-1$, then by $(3.1)$ we have $\varepsilon_{m(\mathbf{b})}=-1$, and hence $b_{m(\mathbf{b})}=-1$. It follows that

$$
\varepsilon_{i}=b_{i}=1, \quad \text { for } i=m(\mathbf{b})+1, m(\mathbf{b})+3, \ldots, M(\mathbf{b})-3, M(\mathbf{b})-1, M(\mathbf{b}) .
$$

It is easy to verify that (3.7) and (3.8) are two contradicting identities. This shows that $a_{m(\mathbf{b})}=-1$ is impossible. Therefore $a_{m(\mathbf{b})}=0$, and $m(\mathbf{b})-m(\mathbf{a})$ must be even by Proposition 3.9.

Before closing the section we prove that the local dimension will be unchanged under the shift operator. Fix any $x \in[-\ell, \ell]$, then $x$ can be obtained by applying the shift operator to any point in $E(x)$ (defined by (1.2)) a finite number of times. 
Consequently, the measure $\mu$ will have the same local dimension value at every point in $E(x)$.

For any $x=\sum_{i=1}^{\infty} \varepsilon_{i} \rho^{i}$, where $\varepsilon_{i}= \pm 1$, define the shift operator $\sigma$ by $\sigma x=$ $\sum_{i=1}^{\infty} \varepsilon_{i+1} \rho^{i}$.

Lemma 3.11. Let $\rho=(\sqrt{5}-1) / 2$. For any $x \in[-\ell, \ell]$, we have $\bar{d}(\mu, x)=\bar{d}(\mu, \sigma x)$ and $\underline{d}(\mu, x)=\underline{d}(\mu, \sigma x)$.

Proof. Let the components of $\varepsilon=\left(\varepsilon_{1}, \varepsilon_{2}, \ldots\right)$ be the coefficients of $x$, and let the components of $\sigma \varepsilon=\left(\varepsilon_{2}, \varepsilon_{3}, \ldots\right)$ be the coefficients of $\sigma x$. Obviously, for any $n$ we have

$$
p_{n}(\sigma \varepsilon) \leq p_{n+1}(\varepsilon) .
$$

On the other hand, any zero element of $\boldsymbol{\varepsilon}^{(\mathbf{n}+\mathbf{1})}=\left(\varepsilon_{1}, \varepsilon_{2}, \ldots, \varepsilon_{n+1}\right)$ is either a zero element contained in $(\sigma \varepsilon)^{(\mathbf{n})}=\left(\varepsilon_{2}, \varepsilon_{3}, \ldots, \varepsilon_{n+1}\right)$ or a zero element containing $\varepsilon_{1}$. By Proposition 3.10, the number of atoms in $\boldsymbol{\varepsilon}^{(\mathbf{n}+\mathbf{1})}$ containing $\varepsilon_{1}$ is less than $n / 2$. So

$$
\mathbb{Z}_{n+1}\left(\varepsilon^{(\mathbf{n}+\mathbf{1})}\right) \leq \mathbb{Z}_{n}\left((\sigma \varepsilon)^{(\mathbf{n})}\right)+n \mathbb{Z}_{n}\left((\sigma \varepsilon)^{(\mathbf{n})}\right) / 2 .
$$

In other words, we have for all $n$

$$
p_{n+1}(\varepsilon) \leq(1+n / 2) p_{n}(\sigma \varepsilon) .
$$

On the other hand, Lemma 3.5 implies that

$$
p_{n}(\sigma \varepsilon) \leq p_{n+1}(\sigma \varepsilon) \leq p_{n}(\sigma \varepsilon)+n p_{n}(\sigma \varepsilon) / 2=(1+n / 2) p_{n}(\sigma \varepsilon) .
$$

This together with (3.9) and (3.10) imply that

$$
\frac{\log p_{n+1}(\sigma \varepsilon)}{n+1} \text { and } \frac{\log p_{n+1}(\varepsilon)}{n+1}
$$

have the same lower and upper limit. The lemma thus follows from (3.3) and Lemma 2.6.

Corollary 3.12. Let $\rho=(\sqrt{5}-1) / 2$. For any $x \in[-\ell, \ell]$, let $E(x)$ be the dense subset of $[-\ell, \ell]$ defined by (1.2). Then $\bar{d}(\mu, z)=\bar{d}(\mu, x)$ and $\underline{d}(\mu, z)=\underline{d}(\mu, x)$ for every $z \in E(x)$.

Remark 3.13. The upper and lower local dimension in Corollary 3.12 cannot be replaced by upper or lower $\alpha$-density. This is justified by the following simple example.

Let $x=-\rho+\sum_{i=2}^{\infty} \rho^{i}$. Then $\sigma x=\sum_{i=1}^{\infty} \rho^{i}$. It is proved in Theorem 2.3 that $\mu$ has a positive $\alpha^{*}$-upper and $\alpha^{*}$-lower density at $\sigma x$, where $\alpha^{*}=-\frac{\log 2}{\log \rho}$. The coefficients of $x$ are the components of $\varepsilon=(-1,1, \ldots, 1, \ldots)$. For every odd integer $n, \boldsymbol{\varepsilon}^{(\mathbf{n})}=(-1,1, \ldots, 1)$ contains the following $(n-1) / 2$ atoms: $(-1,1,1,0, \ldots, 0)$, $(-1,1,0,1,1,0, \ldots, 0), \quad(-1,1,0,1,0,1,1,0, \ldots, 0), \ldots,(-1,1,0,1,0,1, \ldots, 0,1,0$, $1,1)$. From Remark 3.3 we see that $p_{n}(\varepsilon) \rightarrow \infty$, as $n \rightarrow \infty$. It follows that

$$
\lim _{n \rightarrow \infty} \mu_{n}\left(I\left(x,(1+\ell) \rho^{n}\right)\right) / \rho^{n \alpha^{*}} \geq \lim _{n \rightarrow \infty} 2^{-n} \#\left\langle x_{n}\right\rangle / 2^{-n}=\lim _{n \rightarrow \infty} p_{n}(\varepsilon)=\infty .
$$

By Lemma 2.1, we obtain $\lim _{r \rightarrow 0^{+}} \mu(I(x, r)) / r^{\alpha^{*}}=\infty$. 


\section{The minimum value of $d(\mu, x)$}

For any $\varepsilon=\left(\varepsilon_{1}, \varepsilon_{2}, \ldots\right) \in\{-1,1\}^{\infty}$, let $J(\varepsilon)=\left\{n: p_{n}(\varepsilon)>p_{n-1}(\varepsilon), n=\right.$ $2,3, \ldots\}$. If $j \in J(\varepsilon)$ is not the smallest index, denote $j^{\prime}=\max \{n \in J(\varepsilon): n<$ $j$ \}. Based on Lemma 3.5, we can derive the following more concrete recurrence equations and inequalities for computing $p_{n}=p_{n}(\varepsilon)$.

Lemma 4.1. Let $\rho=(\sqrt{5}-1) / 2$, and let $\varepsilon$ be defined as above. For any $j \in J(\varepsilon)$ which is not the smallest index, let $i=j^{\prime}$ and let $q=j-i$. We have

(i) $q \geq 2$.

(ii) Suppose that $q$ is odd. If $q=3$, then $p_{j}=2 p_{i}$. If $q>3$, then $p_{j} \leq(q+1) p_{i} / 2$.

(iii) Suppose that $q$ is even and $\varepsilon_{i}=-\varepsilon_{j}$. If $q=2$, then $p_{j}=p_{i}+p_{i-2}$. If $q>2$, then $p_{j} \leq q p_{i} / 2+p_{i-2}$.

(iv) Suppose that $q$ is even and $\varepsilon_{i}=\varepsilon_{j}$. If $q=2$, then $p_{j}=2 p_{i}-p_{i-2}$; if $q>2$, then $p_{j} \leq(q / 2+1) p_{i}-p_{i-2}$.

Before going through the technical proof, we give two examples for case (iii) as an illustration. Let $\varepsilon$ be defined in such a way that $\varepsilon^{(\mathbf{5})}=(1,-1,-1,1,1)$. Then $\varepsilon^{(\mathbf{5})}$ contains two nontrivial atoms: $\mathbf{a}_{\mathbf{1}}=(1,-1,-1,0,0)$ and $\mathbf{a}_{\mathbf{2}}=(0,0,-1,1,1)$. By Lemma 3.4, $i=3 \in J(\varepsilon), j=5 \in J(\varepsilon)$, and $q=2$. Since $\mathbf{a}_{\mathbf{1}}$ and $\mathbf{a}_{\mathbf{2}}$ cannot form a direct sum, the zero elements of $\varepsilon^{(5)}$ include: the unit zero and $\mathbf{a}_{\mathbf{1}}$ (these two come from the zero elements of $\left.\varepsilon^{(3)}\right)$ and $\mathbf{a}_{2}$ (this one can be considered as the direct sums of $\mathbf{a}_{\mathbf{2}}$ and the zero element of $\boldsymbol{\varepsilon}^{(\mathbf{2})}$, i.e., the unit zero). So $p_{5}=p_{3}+p_{2}=p_{3}+p_{1}$.

If $\varepsilon$ is defined so that $\varepsilon^{(\mathbf{7})}=(1,-1,-1,1,-1,1,1)$, then $\varepsilon^{(\mathbf{7})}$ contains three nontrivial atoms: $\mathbf{a}_{\mathbf{1}}=(1,-1,-1,0, \ldots, 0), \mathbf{a}_{\mathbf{2}}=(0, \ldots, 0,-1,1,1)$ and $\mathbf{a}_{\mathbf{3}}=$ $(0,0,-1,1,0,1,1)$. Note that the final entries of $\mathbf{a}_{2}$ and $\mathbf{a}_{\mathbf{3}}$ are equal, so we have only $i=3 \in J(\varepsilon)$ ad $j=7 \in J(\varepsilon)$. Thus $q=4>2$. Since $\mathbf{a}_{\mathbf{1}}$ and $\mathbf{a}_{\mathbf{3}}$ cannot form a direct sum, the zero elements of $\varepsilon^{(\mathbf{7})}$ come from either the zero elements of $\varepsilon^{(\mathbf{3})}$, or the direct sums of the zero elements of $\varepsilon^{(3)}$ with $\mathbf{a}_{2}$ (total number equals $p_{3}$ ), or the direct sums of the zero elements of $\boldsymbol{\varepsilon}^{(\mathbf{2})}$ with $\mathbf{a}_{\mathbf{3}}$ (total number equals $p_{2}$ ). So $p_{7}=p_{3}+p_{3}+p_{2}=2 p_{3}+p_{1}$. If the fifth entry of $\varepsilon^{(\mathbf{7})}$ is 1 instead of -1 , then $\varepsilon^{(\mathbf{7})}$ contains only $\mathbf{a}_{\mathbf{1}}$ and $\mathbf{a}_{\mathbf{3}}$ as nontrivial atoms and, similarly, $p_{7}=p_{3}+p_{2}<2 p_{3}+p_{1}$.

Proof of Lemma 4.1. As before, let $\varepsilon^{(\mathbf{n})}=\left(\varepsilon_{1}, \ldots, \varepsilon_{n}\right)$. Since $i, j \in J(\varepsilon)$, by Lemma 3.4 and by the structure of atoms, there are two atoms $\mathbf{a}=\left(a_{1}, \ldots, a_{i}\right)$ and $\mathbf{b}=\left(b_{1}, \ldots, b_{j}\right)$ of $\boldsymbol{\varepsilon}^{(\mathbf{i})}$ and $\boldsymbol{\varepsilon}^{(\mathbf{j})}$, respectively, such that

$$
a_{k}=\varepsilon_{k}=\varepsilon_{i} \neq 0, \quad \text { for } k=m(\mathbf{a})+1, m(\mathbf{a})+3, \ldots, i-3, i-1, i,
$$

and

$$
b_{k}=\varepsilon_{k}=\varepsilon_{j} \neq 0, \quad \text { for } k=m(\mathbf{b})+1, m(\mathbf{b})+3, \ldots, j-3, j-1, j .
$$

In particular, $M(\mathbf{a})=i$ and $M(\mathbf{b})=j$.

If $q=1$, then $M(\mathbf{b})-M(\mathbf{a})=1$, so $\mathbf{a}$ and $\mathbf{b}$ cannot be strongly mutually disjoint, which contradicts Proposition 3.10. Therefore $q \geq 2$. This proves (i).

Note that (i) implies that $p_{j-1}=p_{j-2}=p_{i}$.

If $q$ is odd, by Proposition 3.10, $\mathbf{a}$ and $\mathbf{b}$ must be strongly mutually disjoint, so $m(\mathbf{b}) \geq i+1$. If $q=3$, then $m(\mathbf{b})=i+1$ and $\mathbf{b}=\left(0, \ldots, 0, b_{i+1}, b_{i+2}, b_{j}\right)$ is the only atom of $\boldsymbol{\varepsilon}^{(\mathbf{j})}$ whose final entry equals $\varepsilon_{j}$. By Lemma $3.5, p_{j}=p_{j-1}+p_{m(\mathbf{b})-1}=$ $p_{i}+p_{(i+1)-1}=2 p_{i}$. If $q \geq 5$, note that $j-m(\mathbf{b})=M(\mathbf{b})-m(\mathbf{b})$ must be even for $\mathbf{b} \in \mathbb{A}_{j}$ and that $i+1 \leq m(\mathbf{b}) \leq j-2$. Therefore the total number of such $\mathbf{b}$ 's is 
not greater than $[(j-2)-(i+1)] / 2+1=(q-1) / 2$. Applying Lemma 3.5, we get $p_{j} \leq p_{j-1}+(q-1) p_{i} / 2=(q+1) p_{i} / 2$. This proves (ii).

For the rest of the proof we assume that $q$ is even.

Suppose that $\varepsilon_{i}=-\varepsilon_{j}$. We first claim that $m(\mathbf{b}) \geq i$. Actually if $m(\mathbf{b})<i$ $(<j=M(\mathbf{b}))$, then either $i$ or $(i-1)$ is in the set of indices $\{m(\mathbf{b})+1, m(\mathbf{b})+$ $3, \ldots, j-3, j-1\}$. Without loss of generality, assume that $(i-1)$ is in this set. By (4.2), $\varepsilon_{i-1}=b_{i-1}=\varepsilon_{j}$. But (4.1) and the assumption imply that $\varepsilon_{i-1}=\varepsilon_{i}=-\varepsilon_{j}$. The contradiction shows that $m(\mathbf{b}) \geq i$.

If $q=2$, then $\mathbf{b}=\left(0, \ldots, 0, b_{i}, b_{i+1}, b_{j}\right)$ will be the only atom of $\varepsilon^{(\mathbf{j})}$ whose final entry equals $\varepsilon_{j}$. By Lemma $3.5, p_{j}=p_{i}+p_{i-1}=p_{i}+p_{i-2}$. Suppose that $q \geq 4$. Since $i \leq m(\mathbf{b}) \leq j-2$ and $j-m(\mathbf{b})$ must be even, the total number of such b's is not greater than $[(j-2)-i] / 2+1=q / 2$. Among them, at most one satisfies $m(\mathbf{b})=i$, while the rest satisfy $m(\mathbf{b})>i$. This implies that $p_{j} \leq$ $p_{i}+p_{i-1}+(q / 2-1) p_{i}=q p_{i} / 2+p_{i-2}$. This proves (iii).

Suppose that $\varepsilon_{i}=\varepsilon_{j}$. Since $\mathbf{b} \in \mathbb{A}_{j}$, by definition, $b_{m(\mathbf{b})}=-b_{j}=-\varepsilon_{j}$. We first show that

$$
m(\mathbf{b}) \neq i-1 \quad \text { and } \quad m(\mathbf{b}) \neq i .
$$

In fact, if $m(\mathbf{b})=i-1$, by (4.1) and (3.1), it follows that $\varepsilon_{i}=\varepsilon_{i-1}=b_{i-1}=-\varepsilon_{j}$. This contradicts the hypothesis $\varepsilon_{i}=\varepsilon_{j}$. Similarly it can be shown that $m(\mathbf{b}) \neq i$.

Suppose that $q=2$, then $m(\mathbf{b}) \leq j-2=i$. Thus $m(\mathbf{b}) \leq i-2$. If $\mathbf{a}=$ $\left(a_{1}, \ldots, a_{i}\right)$ is an atom satisfying (4.1), by $(3.1), \varepsilon_{i}=\varepsilon_{j}$ implies that $a_{i}=b_{j}$; hence $\left(a_{1}, \ldots, a_{i-1}, 0, b_{j-1}, b_{j}\right)$ is an atom satisfying (4.2). Similarly if $\mathbf{b}=\left(b_{1}, \ldots, b_{j}\right)$ is an atom satisfying (4.2), then $\left(b_{1}, \ldots, b_{j-3}, a_{i}\right)$ is an atom satisfying (4.1). So both $\varepsilon^{(\mathbf{i})}$ and $\varepsilon^{(\mathbf{j})}$ contain the same number of atoms whose final entries equal $\varepsilon_{j}$, denoted respectively by $\mathbf{a}_{n}$ and $\mathbf{b}_{n}$, for $n=1, \ldots, N$. We can actually assume that $m\left(\mathbf{a}_{n}\right)=m\left(\mathbf{b}_{n}\right)$, for $n=1, \ldots, N$. Using Lemma 3.5 we conclude

$$
p_{j}=p_{i}+\sum_{n=1}^{N} p_{m\left(\mathbf{b}_{n}\right)-1}=p_{i}+\sum_{n=1}^{N} p_{m\left(\mathbf{a}_{n}\right)-1}=p_{i}+\left(p_{i}-p_{i-2}\right)=2 p_{i}-p_{i-2} .
$$

Suppose that $q \geq 4$. The b's satisfying (4.2) can be exactly divided into two classes. Class $\mathcal{A}$ consists of those with $m(\mathbf{b}) \leq i-2$ and class $\mathcal{B}$ consists of those with $m(\mathbf{b})>i-2$. First consider the case in which $m(\mathbf{b}) \leq i-2$. Since $j-i$ is even, (4.2) is valid for $k=i-1, i+1, \ldots, j-3, j-1$. So if $\mathbf{a}=\left(a_{1}, \ldots, a_{i}\right)$ is an atom satisfying (4.1), by the same reasons as for the case $q=2,\left(a_{1}, \ldots, a_{i-1}, 0, b_{i+1}, \ldots, b_{j}\right)$ is an atom satisfying (4.2). Similarly if $\mathbf{b}=\left(b_{1}, \ldots, b_{j}\right)$ is an atom satisfying (4.2), then $\left(b_{1}, \ldots, b_{i-1}, a_{i}\right)$ is an atom satisfying (4.1). It follows that

$$
\sum_{\mathbf{b} \in \mathcal{A}} p_{m(\mathbf{b})-1}=\sum_{n=1}^{N} p_{m\left(\mathbf{a}_{n}\right)-1}
$$

where $\mathbf{a}_{n}$, for $n=1, \ldots, N$, are all atoms of $\boldsymbol{\varepsilon}^{(\mathbf{i})}$ satisfying (4.1). If $m(\mathbf{b})>i-2$, then by (4.3), $m(\mathbf{b})>i$. Since both $j-i$ and $j-m(\mathbf{b})$ are even, $m(\mathbf{b})-i$ is even and hence $i+2 \leq m(\mathbf{b}) \leq j-2$. Thus the total number of such b's is not greater 
than $[(j-2)-(i+2)] / 2+1=q / 2-1$. Applying Lemma 3.5, we conclude that

$$
\begin{aligned}
p_{j} & =p_{i}+\sum_{\mathbf{b} \in \mathcal{A}} p_{m(\mathbf{b})-1}+\sum_{\mathbf{b} \in \mathcal{B}} p_{m(\mathbf{b})-1} \leq p_{i}+\sum_{n=1}^{N} p_{m\left(\mathbf{a}_{n}\right)-1}+(q / 2-1) p_{i} \\
& =p_{i}+\left(p_{i}-p_{i-2}\right)+(q / 2-1) p_{i}=(q / 2+1) p_{i}-p_{i-2} .
\end{aligned}
$$

Let $F_{n}$, for $n=0,1, \ldots$, be the Fibonacci sequence, i.e., $F_{1}=F_{0}=1$ and $F_{n+1}=F_{n}+F_{n-1}$, for $n=1,2, \ldots$ It is known that the solution to this recurrence relation is

$$
F_{n}=\left[\delta^{-(n+1)}-(-\delta)^{n+1}\right] / \sqrt{5}, \quad \text { where } \delta=(\sqrt{5}-1) / 2 .
$$

Lemma 4.2. Let $\rho=(\sqrt{5}-1) / 2$, and let $\varepsilon=\left(\varepsilon_{1}, \varepsilon_{2}, \ldots\right) \in\{-1,1\}^{\infty}$. Then for $j \in J(\varepsilon)$ and for $k \geq 1$, the following hold:

(i) If $\varepsilon_{j+2 i}=\varepsilon_{j+2 i-1}=(-1)^{i} \varepsilon_{j}$, for $i=1, \ldots, k$, then $p_{j+2 k}=F_{k} p_{j}+F_{k-1} p_{j-2}$.

(ii) If $\varepsilon_{j+2 i+1}=\varepsilon_{j+2 i}=(-1)^{i} \varepsilon_{j+1}$, for $i=1, \ldots, k$, then $p_{j+2 k+1}=F_{k+1} p_{j}$.

(iii) If $\varepsilon_{j+i+1}=(-1)^{i} \varepsilon_{j+1}$, for $i=1,2, \ldots, 2 k-1$, and $\varepsilon_{j+2 k+1}=\varepsilon_{j+2 k}$, then $p_{j+2 k+1}=(k+1) p_{j}$.

Moreover, (ii) and (iii) are still valid for $j=0$ if we define $p_{0}=1$.

Proof. We first show (i). It is easy to see that $(j+2 i) \in J(\varepsilon)$ for $i=1, \ldots, k$. Repeatedly applying (iii) of Lemma 4.1 gives $p_{j+2}=p_{j}+p_{j-2}=F_{1} p_{j}+F_{0} p_{j-2}$, $p_{j+4}=p_{j+2}+p_{j}=F_{2} p_{j}+F_{1} p_{j-2}, \ldots, p_{j+2 k}=p_{j+2 k-2}+p_{j+2 k-4}=F_{k} p_{j}+$ $F_{k-1} p_{j-2}$.

For (ii), since $j \in J(\varepsilon)$, by (i) of Lemma $4.1,(j+1) \notin J(\varepsilon)$. Clearly $(j+2 i+1) \in$ $J(\varepsilon)$ for $i=1, \ldots, k$. Applying (ii) and (iii) of Lemma 4.1, we obtain $p_{j+3}=2 p_{j}=$ $F_{2} p_{j}$ (if $j=0$, then $\left.p_{3}=2=F_{2} p_{0}\right), p_{j+5}=p_{j+3}+p_{j}=F_{3} p_{j}, \ldots, p_{j+2 k+1}=$ $p_{j+2 k-1}+p_{j+2 k-3}=F_{k+1} p_{j}$.

To show (iii), note that the final two nonzero entries of any atom must be equal, therefore $i \notin J(\varepsilon)$ for $i=j+1, j+2, \ldots, j+2 k$, and clearly $(j+2 k+1) \in J(\varepsilon)$. An argument similar to the one used in the proof of (ii) of Lemma 4.1 shows that $\left(\varepsilon_{j+1}, \varepsilon_{j+2}, \ldots, \varepsilon_{j+2 k+1}\right)$ contains exactly $k$ atoms whose final entries equal $\varepsilon_{j+2 k+1}$, so $p_{j+2 k+1}=p_{j}+k p_{j}=(k+1) p_{j}$.

Lemma 4.3. Let $\rho=(\sqrt{5}-1) / 2$. For any $\boldsymbol{\omega} \in\{-1,1\}^{\infty}$, there exists $\boldsymbol{\tau}=$ $\left(\tau_{1}, \tau_{2}, \ldots\right) \in\{-1,1\}^{\infty}$ having the following two properties:

(a) The smallest index of $J(\boldsymbol{\tau})$ is 3. For all other $j \in J(\boldsymbol{\tau})$, we have $j-j^{\prime}=2$ or 3 and $\tau_{j}=-\tau_{j^{\prime}}$.

(b) $p_{i}(\boldsymbol{\tau}) \geq p_{i}(\boldsymbol{\omega})$ for all $i=1,2, \ldots$.

Proof. We will use the pattern (i) or (ii) of Lemma 4.2 to define $\left(\tau_{1}, \tau_{2}, \ldots\right)$. Let $\tau_{1}=1$. If the smallest index $m$ in $J(\boldsymbol{\omega})$ equals $2 k+1(\geq 3)$, then let $\tau_{2 i+1}=$ $\tau_{2 i}=(-1)^{i}$, for $i=1, \ldots, k$ (if $m=2 k$, let $\tau_{2}=1, \tau_{2 i+2}=\tau_{2 i+1}=(-1)^{i}$, for $i=1, \ldots, k-1)$. In general, suppose that $\left(\tau_{1}, \ldots, \tau_{j^{\prime}}\right)$ is defined for any $j \in J(\boldsymbol{\omega})$. If $j-j^{\prime}=2 n+1$, we let $\tau_{j^{\prime}+1}=\tau_{j^{\prime}}$ and let $\tau_{j^{\prime}+2 i+1}=\tau_{j^{\prime}+2 i}=(-1)^{i} \tau_{j^{\prime}+1}$ for $i=1, \ldots, n$ (if $j-j^{\prime}=2 n$, we let $\tau_{j^{\prime}+2 i}=\tau_{j^{\prime}+2 i-1}=(-1)^{i} \tau_{j^{\prime}}$ for $i=1, \ldots, n$ ). Define $\boldsymbol{\tau}=\left(\tau_{1}, \tau_{2}, \ldots\right)$. From the construction of $\boldsymbol{\tau}$, we see that the smallest index of $J(\boldsymbol{\tau})$ is 4 if $m$ is even and is 3 if $m$ is odd. In addition, $\tau_{j}=-\tau_{j^{\prime}}$ and $j-j^{\prime} \leq 3$ for all other $j \in J(\boldsymbol{\tau})$. So (a) holds except possibly when the smallest index of $J(\boldsymbol{\tau})$ is 4 . In this case, let $\sigma \boldsymbol{\tau}=\left(\tau_{2}, \tau_{3}, \ldots\right)$ be the shift of $\boldsymbol{\tau}$. Then $\sigma \boldsymbol{\tau}$ satisfies (a) and $p_{i+1}(\sigma \boldsymbol{\tau}) \geq p_{i}(\sigma \boldsymbol{\tau})=p_{i+1}(\boldsymbol{\tau})$ for all $i$. Hence, if $\boldsymbol{\tau}$ satisfies (b), so does $\sigma \boldsymbol{\tau}$. 
We show (b) by induction. If $i<m$, then $p_{i}(\boldsymbol{\tau}) \geq 1=p_{i}(\boldsymbol{\omega})$. Consider $i=m$. Suppose that $m=2 k+1$. By (ii) of Lemma 4.2, $p_{m}(\boldsymbol{\tau})=F_{k+1}$. Using an argument similar to that in the proof of (ii) in Lemma 4.1, we have $p_{m}(\boldsymbol{\omega}) \leq k+1$ (if $m=2 k$, then $p_{m}(\boldsymbol{\tau})=F_{k}$ and $p_{m}(\boldsymbol{\omega}) \leq k$ ). So $p_{m}(\boldsymbol{\tau}) \geq p_{m}(\boldsymbol{\omega})$. Thus (b) is true for all $i \leq m$. Suppose that (b) is true for all $i \leq n-1$, where $n-1 \geq m$. If $n \notin J(\boldsymbol{\omega})$, by the induction hypothesis, $p_{n}(\boldsymbol{\omega})=p_{n-1}(\boldsymbol{\omega}) \leq p_{n-1}(\boldsymbol{\tau}) \leq p_{n}(\boldsymbol{\tau})$, and (b) follows. If $n \in J(\boldsymbol{\omega})$, let $n^{\prime}=\max \{k \in J(\boldsymbol{\omega}): k<n\}$. Since $J(\boldsymbol{\omega}) \subseteq J(\boldsymbol{\tau})$ by the construction of $\boldsymbol{\tau}$, we have $n \in J(\boldsymbol{\tau})$ and $n^{\prime} \in J(\boldsymbol{\tau})$.

Case 1. $n-n^{\prime}=2 j+1$. By applying (ii) of Lemma 4.2, the induction hypothesis and (ii) of Lemma 4.1 we obtain

$$
p_{n}(\boldsymbol{\tau})=F_{j+1} p_{n^{\prime}}(\boldsymbol{\tau}) \geq(j+1) p_{n^{\prime}}(\boldsymbol{\tau}) \geq(j+1) p_{n^{\prime}}(\boldsymbol{\omega}) \geq p_{n}(\boldsymbol{\omega}) .
$$

Case 2. $n-n^{\prime}=2 j$. Part (i) of Lemma 4.2 implies

$$
p_{n}(\boldsymbol{\tau})=F_{j} p_{n^{\prime}}(\boldsymbol{\tau})+F_{j-1} p_{n^{\prime}-2}(\boldsymbol{\tau}) .
$$

On the other hand, applying (iii), (iv) of Lemma 4.1, we have

$$
p_{n}(\boldsymbol{\omega}) \leq \max \left\{j p_{n^{\prime}}(\boldsymbol{\omega})+p_{n^{\prime}-2}(\boldsymbol{\omega}),(j+1) p_{n^{\prime}}(\boldsymbol{\omega})-p_{n^{\prime}-2}(\boldsymbol{\omega})\right\} .
$$

It is clear that $p_{n}(\boldsymbol{\tau})$ is greater than the first term inside the braces, so we only need to show that $p_{n}(\boldsymbol{\tau}) \geq t$, where $t=(j+1) p_{n^{\prime}}(\boldsymbol{\omega})-p_{n^{\prime}-2}(\boldsymbol{\omega})$ is the second term inside the braces. This can be proved by two subcases.

Subcase 1. $n^{\prime}$ is the smallest index in $J(\boldsymbol{\omega})$, i.e., $n^{\prime}=m$. Then

$$
t=(j+1) p_{m}(\boldsymbol{\omega})-1 \leq \begin{cases}(j+1)(k+1)-1, & \text { if } m=2 k+1, \\ (j+1) k-1, & \text { if } m=2 k\end{cases}
$$

and

$$
p_{n}(\boldsymbol{\tau})=F_{j} p_{m}(\boldsymbol{\tau})+F_{j-1} p_{m-2}(\boldsymbol{\tau})= \begin{cases}F_{j} F_{k+1}+F_{j-1} F_{k}, & \text { if } m=2 k+1, \\ F_{j} F_{k}+F_{j-1} F_{k-1}, & \text { if } m=2 k .\end{cases}
$$

A routine check shows that $p_{n}(\boldsymbol{\tau}) \geq t$ for all positive integers $j$ and $k$.

Subcase 2. $n^{\prime}>m$. By (a) we see that either $\left(n^{\prime}-2\right) \in J(\boldsymbol{\tau})$ or $\left(n^{\prime}-3\right) \in J(\boldsymbol{\tau})$. Using Lemma 4.1, if $\left(n^{\prime}-3\right) \in J(\boldsymbol{\tau})$, then $p_{n^{\prime}}(\boldsymbol{\tau})=2 p_{n^{\prime}-3}(\boldsymbol{\tau})=2 p_{n^{\prime}-2}(\boldsymbol{\tau})$; and if $\left(n^{\prime}-2\right) \in J(\boldsymbol{\tau})$, then $p_{n^{\prime}}(\boldsymbol{\tau})=p_{n^{\prime}-2}(\boldsymbol{\tau})+p_{n^{\prime}-4}(\boldsymbol{\tau}) \leq 2 p_{n^{\prime}-2}(\boldsymbol{\tau})$. In any case, we obtain $p_{n^{\prime}-2}(\boldsymbol{\tau}) \geq p_{n^{\prime}}(\boldsymbol{\tau})-p_{n^{\prime}-2}(\boldsymbol{\tau})$. Therefore

$$
p_{n}(\boldsymbol{\tau}) \geq j p_{n^{\prime}}(\boldsymbol{\tau})+p_{n^{\prime}-2}(\boldsymbol{\tau}) \geq(j+1) p_{n^{\prime}}(\boldsymbol{\tau})-p_{n^{\prime}-2}(\boldsymbol{\tau}) \geq t
$$

Theorem 4.4. Let $\rho=(\sqrt{5}-1) / 2$. The minimum value of the lower local dimensions $\underline{d}(\mu, x), x \in[-\ell, \ell]$, is given by $\alpha_{*}=-1 / 2-\frac{\log 2}{\log \rho} \approx 0.9404$. Furthermore, $0<\underline{\lim }_{r \rightarrow 0^{+}} \mu(I(z, r)) / r^{\alpha_{*}} \leq \varlimsup_{r \rightarrow 0^{+}} \mu(I(z, r)) / r^{\alpha_{*}}<\infty$ for every point $z$ in the dense subset $E\left(\rho^{4} /\left(1-\rho^{4}\right)\right)$ of $[-\ell, \ell]$, where $E\left(\rho^{4} /\left(1-\rho^{4}\right)\right)$ is defined by $(1.2)$.

Proof. Let $\varepsilon_{1}=1$, and let $\varepsilon_{2 k}=\varepsilon_{2 k+1}=(-1)^{k}$, for $k=1,2, \ldots$ Define $\varepsilon=$ $\left(\varepsilon_{1}, \varepsilon_{2}, \ldots\right)$ and define

$$
z=\sum_{i=1}^{\infty} \varepsilon_{i} \rho^{i}\left(=\sum_{i=1}^{\infty} \rho^{4 i}=\rho^{4} /\left(1-\rho^{4}\right)\right) .
$$

We will show that the point $z$ and the sequence $n_{k}=2 k+1, k=1,2, \ldots$ satisfy (i), (ii) and (iii) of Lemma 2.4. Clearly for $k=1,2 \ldots,(2 k+1) \in J(\varepsilon)$ and by (ii) 
of Lemma $4.2, p_{2 k+1}(\varepsilon)=F_{k+1}$. We first show that $z$ satisfies (ii) of Lemma 2.4. By (3.3), it suffices to show that for any $\boldsymbol{\omega} \in\{-1,1\}^{\infty}$ we have

$$
p_{2 k+1}(\boldsymbol{\omega}) \leq p_{2 k+1}(\varepsilon), \quad \text { for } k=1,2, \ldots .
$$

Let $\boldsymbol{\tau}=\left(\tau_{1}, \tau_{2}, \ldots\right) \in\{-1,1\}^{\infty}$, depending on $\boldsymbol{\omega}$, be defined as in Lemma 4.3. Then (4.5) will follow if we can show that

$$
p_{2 k+1}(\boldsymbol{\tau}) \leq F_{k+1}, \quad \text { for } k=1,2, \ldots .
$$

Since the smallest index of $J(\boldsymbol{\tau})$ is 3 , it follows that $p_{3}(\boldsymbol{\tau})=2=F_{2}$. Let $k>1$ and consider the sequence $\left(\tau_{4}, \tau_{5}, \ldots, \tau_{2 k+1}\right)$. If $i-i^{\prime}=2$ for all $i \in J(\boldsymbol{\tau})$ with $5 \leq i \leq 2 k+1$, then (4.6) follows from (ii) of Lemma 4.2. Otherwise, by (a) of Lemma 4.3 , there are some indices $i \in J(\boldsymbol{\tau})$ for which $i-i^{\prime}=3$. Without loss of generality, assume that the total number of such indices is even (the proof is similar if this number is odd). Actually we can further assume that this even number is two, and that one such index equals 6 and the other one equals $2 k+1$; the other cases can be easily proven by induction. Under these assumptions, $\left(\tau_{7}, \tau_{8}, \ldots, \tau_{2 k-2}\right)$ satisfies $i-i^{\prime}=2$ for all $i \in J(\boldsymbol{\tau})$ with $8 \leq i \leq 2 k-2$. Using (i) of Lemma 4.2 and (ii) of Lemma 4.1, we get

$$
p_{2 k-2}(\boldsymbol{\tau})=F_{(2 k-8) / 2} p_{6}(\boldsymbol{\tau})+F_{(2 k-8) / 2-1} p_{3}(\boldsymbol{\tau})=\left(2 F_{k-4}+F_{k-5}\right) p_{3}(\boldsymbol{\tau})=2 F_{k-2} .
$$

Therefore

$$
p_{2 k+1}(\boldsymbol{\tau})=2 p_{2 k-2}(\boldsymbol{\tau})=4 F_{k-2} \leq F_{k+1} .
$$

This proves (4.6). On the other hand, if $\alpha_{*}=-1 / 2-\frac{\log 2}{\log \rho}$, then $2 \rho^{\alpha_{*}}=\rho^{-1 / 2}$. By using (4.4) and noting that $\delta=\rho$, we obtain

$$
p_{2 k+1}(\varepsilon) /\left(2 \rho^{\alpha_{*}}\right)^{2 k+1}=F_{k+1} / \rho^{-k-1 / 2}=\left[\delta^{-(k+2)}-(-\delta)^{k+2}\right] / \sqrt{5} \delta^{-k-1 / 2} .
$$

Hence $0<\lim _{k \rightarrow \infty} p_{2 k+1}(\varepsilon) /\left(2 \rho^{\alpha_{*}}\right)^{2 k+1}<\infty$. By applying (3.3) and Lemma 2.4, it follows that

$$
0<\varliminf_{r \rightarrow 0^{+}} \mu(I(z, r)) / r^{\alpha_{*}} \leq \varlimsup_{r \rightarrow 0^{+}} \mu(I(z, r)) / r^{\alpha_{*}}<\infty
$$

and that $\alpha_{*}$ is the minimum value of the lower local dimensions. Now let $y=$ $\sum_{i=1}^{\infty} \omega_{i} \rho^{i} \in E\left(\rho^{4} /\left(1-\rho^{4}\right)\right)$. By (1.2), there exists a fixed integer $N$ such that $\omega_{N+i}=\varepsilon_{i}$ for all $i=1,2, \ldots$. Applying the above argument, it is not hard to verify that conditions (i)-(iii) of Lemma 2.4 are all satisfied by $y$ and the sequence $N+2 k+$ $1, k=1,2, \ldots$ Therefore $0 \leq \underline{\lim }_{r \rightarrow 0^{+}} \mu(I(y, r)) / r^{\alpha_{*}} \leq \varlimsup_{r \rightarrow 0^{+}} \mu(I(y, r)) / r^{\alpha_{*}}<$ $\infty$.

By using the atom decomposition of a zero element (Remark 3.8) and the arguments of this section, Theorem 4.4 can be generalized to the case in which $\rho$ is a root of (1.1). In fact, let $\varepsilon_{1}=1, \varepsilon_{s k+2}=\varepsilon_{s k+3}=\cdots=\varepsilon_{s k+(s+1)}=(-1)^{k+1}$, for $k=0,1, \ldots$ Define $z=\sum_{i=1}^{\infty} \varepsilon_{i} \rho^{i}\left(=\left(\rho^{s+2}+\rho^{s+3}+\cdots+\rho^{2 s}\right)+\left(\rho^{3 s+2}+\rho^{3 s+3}+\right.\right.$ $\left.\left.\cdots+\rho^{4 s}\right)+\left(\rho^{5 s+2}+\rho^{5 s+3}+\cdots+\rho^{6 s}\right)+\cdots=\left(\rho^{s+2}+\rho^{s+3}+\cdots+\rho^{2 s}\right) /\left(1-\rho^{2 s}\right)\right)$. Then $\#\left\langle z_{s k+1}\right\rangle=F_{k+1} \geq \#\left\langle x_{s k+1}\right\rangle$ for any $x \in[-\ell, \ell]$ and for any series representation of $x$. Also for $\alpha_{*}=-\frac{\log \delta}{s \log \rho}-\frac{\log 2}{\log \rho}$, where $\delta=(\sqrt{5}-1) / 2$, we have $2 \rho^{\alpha_{*}}=\delta^{-1 / s}$. This implies $0<\lim _{k \rightarrow \infty} \#\left\langle z_{s k+1}\right\rangle /\left(2 \rho^{\alpha_{*}}\right)^{s k+1}<\infty$. Hence we have proved the following: 
Theorem 4.5. If $\rho$ is a root of (1.1), then the minimum value of the lower local dimensions $\underline{d}(\mu, x), x \in[-\ell, \ell]$, is given by $\alpha_{*}=-\frac{\log \delta}{s \log \rho}-\frac{\log 2}{\log \rho}$, where $\delta=$ $(\sqrt{5}-1) / 2$. Furthermore, $0<\underline{\lim }_{r \rightarrow 0^{+}} \mu(I(z, r)) / r^{\alpha_{*}} \leq \varlimsup_{r \rightarrow 0^{+}} \mu(I(z, r)) / r^{\alpha_{*}}<$ $\infty$ for every point $z$ in the dense subset $E\left(\left(\rho^{s+2}+\rho^{s+3}+\cdots+\rho^{2 s}\right) /\left(1-\rho^{2 s}\right)\right)$ (defined by $(1.2))$ of $[-\ell, \ell]$.

\section{Other values of $d(\mu, x)$}

For $z=\sum_{i=1}^{\infty} \varepsilon_{i} \rho^{i}$, where $\varepsilon_{i}=(-1)^{i}$, it follows that $\#\left\langle z_{n}\right\rangle=1$ for all $n$. Thus $d(\mu, z)=\alpha^{*}$ (Theorem 2.3). If $\varepsilon_{2 i}=\varepsilon_{2 i+1}=(-1)^{i}$, then $d(\mu, z)=\alpha_{*}$ (Theorem 4.4). In this section, we will suitably arrange the two patterns to define $\left(\varepsilon_{1}, \varepsilon_{2}, \ldots\right)$ so that $d(\mu, z)=\alpha$ for any $\alpha_{*} \leq \alpha \leq \alpha^{*}$. On the other hand, we also give other arrangements so that $\underline{d}(\mu, z)<\alpha_{1}<\alpha_{2}<\bar{d}(\mu, z)$ for any $\alpha_{*}<\alpha_{1}<$ $\alpha_{2}<\alpha^{*}$.

Lemma 5.1. Let $\left(u_{1}, u_{2}, \ldots\right)$ and $\left(v_{1}, v_{2}, \ldots\right)$ be two sequences of real numbers such that $v_{i} \geq 0$ for all $i$ and $\sum_{i=1}^{\infty} v_{i}=\infty$. If $\lim _{i \rightarrow \infty} u_{i} / v_{i}=L$, then

$$
\lim _{i \rightarrow \infty}\left(\sum_{j=1}^{i} u_{j}\right) /\left(\sum_{j=1}^{i} v_{j}\right)=L .
$$

Proof. The lemma can be easily proven by a standard $\varepsilon-N$ argument.

Lemma 5.2. Let $C>0$ be any constant and let $\theta \in(0, C)$. Then there is an integer $j(\theta)$ such that for every $j \geq j(\theta)$ there exist a pair of odd integers $u_{j}>1$ and $v_{j}>1$ such that $u_{j}+v_{j}=2 j$ and

$$
\left|\frac{\log u_{j}+C v_{j}}{u_{j}+v_{j}}-\theta\right| \leq 2(1+C) / j .
$$

Proof. Choose $j(\theta)$ such that $(\log 2 j) / 2 j<\theta<C(1-1 / 2 j)$ for all $j \geq j(\theta)$. Now $f_{j}(x)=(\log x+C(2 j-x)) / 2 j$, for $j \geq j(\theta)$, is a continuous function with $f_{j}(1)=C(1-1 / 2 j)$ and $f_{j}(2 j)=(\log 2 j) / 2 j$, so there is $x_{j} \in[1,2 j]$ such that $f_{j}\left(x_{j}\right)=\theta$. Let $u_{j} \in[3,2 j-3]$ be an odd integer satisfying $\left|u_{j}-x_{j}\right| \leq 4$ and let $v_{j}=2 j-u_{j}$. Then $u_{j}>1$ and $v_{j}>1$. We have

$$
\begin{aligned}
\left|\frac{\log u_{j}+C v_{j}}{u_{j}+v_{j}}-\theta\right| & =\left|f_{j}\left(u_{j}\right)-\theta\right|=\left|f_{j}\left(u_{j}\right)-f_{j}\left(x_{j}\right)\right| \\
& =\left|\log u_{j}-\log x_{j}+C\left(x_{j}-u_{j}\right)\right| / 2 j \\
& \leq(1+C)\left|x_{j}-u_{j}\right| / 2 j \\
& \quad(\text { since }|\log u-\log v| \leq|u-v| \text { for } u, v \geq 1) \\
& \leq 2(1+C) / j .
\end{aligned}
$$

Theorem 5.3. Let $\rho=(\sqrt{5}-1) / 2, \alpha_{*}=-1 / 2-\frac{\log 2}{\log \rho}$ and $\alpha^{*}=-\frac{\log 2}{\log \rho}$.

(i) For every $\alpha_{*} \leq \alpha \leq \alpha^{*}$, there exists $z(\alpha) \in[-\ell, \ell]$ such that $d(\mu, z)=\alpha$ for every point $z$ in the dense subset $E(z(\alpha))$ of $[-\ell, \ell]$, where $E(z(\alpha))$ is defined by $(1.2)$.

(ii) For any $\alpha_{*}<\alpha_{1}<\alpha_{2}<\alpha^{*}$, there exists $z\left(\alpha_{1}, \alpha_{2}\right) \in[-\ell, \ell]$ such that $\underline{d}(\mu, z)<\alpha_{1}<\alpha_{2}<\bar{d}(\mu, z)$ for every point $z$ in the dense subset $E\left(z\left(\alpha_{1}, \alpha_{2}\right)\right)$ of $[-\ell, \ell]$, where $E\left(z\left(\alpha_{1}, \alpha_{2}\right)\right)$ is defined by $(1.2)$. 
Proof. By Corollary 3.12 it suffices to show, respectively, that (i) and (ii) of the theorem are true for some point $z \in[-\ell, \ell]$. Let $k_{i}$, for $i=1,2, \ldots$, be a sequence of odd integers $(\geq 3)$ which will be determined later. Let $n_{0}=0$. For $i=1,2, \ldots$, let

$$
n_{i}=\sum_{j=1}^{i} k_{j} .
$$

Define a sequence $\varepsilon=\left(\varepsilon_{1}, \varepsilon_{2}, \ldots\right) \in\{-1,1\}^{\infty}$ as follows. For $i=0,1, \ldots$, fixed $\varepsilon_{n_{i}+1}=1$. If $i$ is even (including 0 ), then let $\left(\varepsilon_{n_{i}+1}, \varepsilon_{n_{i}+2}, \ldots, \varepsilon_{n_{i}+k_{i+1}}\right)$ have the pattern as in (ii) of Lemma 4.2, i.e., let $\varepsilon_{n_{i}+2 j+1}=\varepsilon_{n_{i}+2 j}=(-1)^{j}$, for $j=$ $1, \ldots,\left(k_{i+1}-1\right) / 2$. If $i$ is odd, then let it have the pattern as in (iii) of Lemma 4.2, i.e., let $\varepsilon_{n_{i}+j+1}=(-1)^{j}$, for $j=1, \ldots, k_{i+1}-2$, and let $\varepsilon_{n_{i}+k_{i+1}}=\varepsilon_{n_{i}+k_{i+1}-1}$. Define

$$
z=\sum_{i=1}^{\infty} \varepsilon_{i} \rho^{i}
$$

It is clear that $n_{i} \in J(\varepsilon)$, for $i=1,2, \ldots$ Applying (ii) and (iii) of Lemma 4.2, we conclude that $p_{n_{1}}(\varepsilon)=F_{\left(k_{1}+1\right) / 2}$ and $p_{n_{i}}(\varepsilon)=F_{\left(k_{i}+1\right) / 2} p_{n_{i-1}}(\varepsilon)$ if $i>1$ is odd, and $p_{n_{i}}(\varepsilon)=\left(k_{i}+1\right) p_{n_{i-1}}(\varepsilon) / 2$ if $i$ is even. So, for $i=1,2, \ldots$, we have

$$
p_{n_{2 i-1}}(\varepsilon)=p_{n_{1}}(\varepsilon) \prod_{j=2}^{i} F_{\left(k_{2 j-1}+1\right) / 2}\left(k_{2 j-2}+1\right) / 2,
$$

and

$$
p_{n_{2 i}}(\varepsilon)=\prod_{j=1}^{i} F_{\left(k_{2 j-1}+1\right) / 2}\left(k_{2 j}+1\right) / 2 .
$$

We first show (i). Without loss of generality, assume that $\alpha_{*}<\alpha<\alpha^{*}$. There exists a $\theta \in(0,-(\log \delta) / 2)$, where $\delta=\rho$, such that $(\theta-\log 2) / \log \rho=\alpha$. Let $j(\theta)$, $u_{j}$ and $v_{j}$, for $j \geq j(\theta)$, be determined as in Lemma 5.2 with $C=-(\log \delta) / 2$, and let $k_{2 j-1}=v_{j}, k_{2 j}=u_{j}$ for $j \geq j(\theta)$. If $j<j(\theta)$ then let $k_{2 j-1}$ and $k_{2 j}$ be any odd integers so that $k_{2 j}+k_{2 j-1} \geq 2 j$. Then we have

$$
\lim _{i \rightarrow \infty} i / n_{2 i}=\lim _{i \rightarrow \infty} i / \sum_{j=1}^{i}\left(k_{2 j}+k_{2 j-1}\right) \leq \lim _{i \rightarrow \infty} i / \sum_{j=1}^{i} 2 j=0 .
$$

From (5.3), (5.4) and (4.4) we have

$$
\begin{aligned}
\lim _{i \rightarrow \infty} \frac{\log \#\left\langle z_{n_{2 i}}\right\rangle}{n_{2 i}} & =\lim _{i \rightarrow \infty} \frac{\log p_{n_{2 i}}(\varepsilon)}{n_{2 i}} \\
& =\lim _{i \rightarrow \infty} \frac{\sum_{j=1}^{i}\left[\log k_{2 j}-(\log \delta) k_{2 j-1} / 2\right]}{\sum_{j=1}^{i}\left(k_{2 j}+k_{2 j-1)}\right.} .
\end{aligned}
$$

Replacing the constant $C$ with $-(\log \delta) / 2$ in Lemma 5.2 , we get

$$
\lim _{j \rightarrow \infty}\left[\log k_{2 j}-(\log \delta) k_{2 j-1} / 2\right] /\left(k_{2 j}+k_{2 j-1}\right)=\theta .
$$

This, together with Lemma 5.1, implies that the limit in (5.5) equals $\theta$. On the other hand, by (5.4), $\lim _{i \rightarrow \infty} n_{2 i} / n_{2(i-1)}=\lim _{i \rightarrow \infty}\left(1+2 i / n_{2(i-1)}\right)=1$. Applying Corollary 2.7, we obtain $d(\mu, z)=(\theta-\log 2) / \log \rho=\alpha$. 
We now show (ii). For $i=1,2$, there exist $\theta_{i} \in(0,-(\log \delta) / 2)$ such that $\left(\theta_{i}-\right.$ $\log 2) / \log \rho=\alpha_{i}$. Notice that $\theta_{2}<\theta_{1}$ since $\log \rho<0$. Choose an odd integer $m$ $(\geq 3)$ satisfying $-(\log \delta) / 2(m+1)<\theta_{2}<\theta_{1}<-m(\log \delta) / 2(m+1)$. Let $z$ be defined by (5.1) such that $k_{j}=m^{j}$ for $j=1,2, \ldots$ By (5.3) we have

$$
\begin{aligned}
\varliminf_{n \rightarrow \infty} \frac{\log \#\left\langle z_{n}\right\rangle}{n} & =\varliminf_{n \rightarrow \infty} \frac{\log p_{n}(\varepsilon)}{n} \leq \lim _{i \rightarrow \infty} \frac{\log p_{n_{2 i}}(\varepsilon)}{n_{2 i}} \\
& =\lim _{i \rightarrow \infty} \frac{\sum_{j=1}^{i}\left[\log m^{2 j}-m^{2 j-1}(\log \delta) / 2\right]}{\sum_{j=1}^{2 i} m^{j}} \\
& =\frac{-\log \delta}{2} \lim _{i \rightarrow \infty} \frac{\sum_{j=1}^{i} m^{2 j-1}}{\sum_{j=1}^{2 i} m^{j}} \\
& =-(\log \delta) / 2(m+1)<\theta_{2} .
\end{aligned}
$$

Similarly, (5.2) implies that

$$
\begin{aligned}
\varlimsup_{n \rightarrow \infty} \frac{\log \#\left\langle z_{n}\right\rangle}{n} & \geq \lim _{i \rightarrow \infty} \frac{\log p_{n_{2 i-1}}(\varepsilon)}{n_{2 i-1}} \\
& =\frac{-\log \delta}{2} \lim _{i \rightarrow \infty} \frac{\sum_{j=2}^{i} m^{2 j-1}}{\sum_{j=2}^{2 i-1} m^{j}} \\
& =-m(\log \delta) / 2(m+1)>\theta_{1} .
\end{aligned}
$$

By using Lemma 2.6, we obtain $\underline{d}(\mu, z)<\alpha_{1}<\alpha_{2}<\bar{d}(\mu, z)$.

Remark 5.4. It can be verified that Theorem 5.3 remains valid when $\rho$ is a root of (1.1).

Finally, we give a computation for the local dimension at $x=0$. Represent $x$ as $\sum_{i=1}^{\infty} \varepsilon_{i} \rho^{i}$ with coefficients $\varepsilon_{i}= \pm 1$ chosen as

$$
\varepsilon=(\underline{1,-1,-1}, \underline{1,-1,-1}, \underline{1,-1,-1}, \ldots, \underline{1,-1,-1}, \ldots) .
$$

Here every underlined triple corresponds to an atom. By Lemma 3.4, $3 i \in J(\varepsilon)$ for all $i$. Repeatedly using (ii) of Lemma 4.1 , we obtain $\#\left\langle x_{3 n}\right\rangle=p_{3 n}(\varepsilon)=2^{n}$. So

$$
\lim _{n \rightarrow \infty} \frac{\log \#\left\langle x_{3 n}\right\rangle}{3 n}=\frac{\log 2}{3} .
$$

By using Corollary 2.7, we have $d(\mu, x)=-(2 \log 2) /(3 \log \rho) \approx 0.9603$. By Corollary 3.12 , this dimension value is valid for all points in the set $E(0)$ defined by $(1.2)$.

\section{ACKNOWLEDGMENTS}

Part of the work was supported by a research grant from University of WisconsinGreen Bay. The author would like to express his deep gratitude to K.-S. Lau for many valuable and stimulating discussions and suggestions. He thanks the nonanonymous referee, R. S. Strichartz, for his careful reading and many constructive remarks, which have made this paper a stronger contribution. He thanks R. B. Wenger for his help in revising the whole manuscript and thanks A. Ho for providing the picture of the measure. 


\section{REFERENCES}

[AZ] J. C. Alexander and D. Zagier, The entropy of a certain infinitely convolved Bernoulli measure, J. London Math. Soc. (2) 44 (1991), 121-134. MR 92g:28035

[AY] J. C. Alexander and J. A. Yorke, Fat baker's transformations, Ergod. Theory \& Dynam. Systems 4 (1984), 1-23. MR 86c:58090

[BDGPS] M. J. Bertin, A. Decomps-Guilloux, M. Grandet-Hugot, M. Pathiaux-Delefosse, and J. P. Schreiber, Pisot and Salem numbers, Birkhäuser-Verlag, Basel, 1992. MR 93k:11095

[E] P. Erdös, On the smoothness properties of a family of Bernoulli convolutions, Amer. J. Math. 62 (1940), 180-186. MR 1:139e

[F] K. J. Falconer, Fractal geometry, mathematical foundations and applications, Wiley, 1990. MR 92j:28008

[G] A. M. Garsia, Arithmetic properties of Bernoulli convolutions, Trans. Amer. Math. Soc. 102 (1962), 409-432. MR 25:1409

[GH] J. S. Geronimo and D. P. Hardin, An exact formula for the measure dimension associated with a class of piecewise linear maps, Constr. Approx. 5 (1989), 89-98. MR 90d: 58076

[JW] B. Jessen and A. Wintner, Distribution functions and the Riemann zeta function, Trans. Amer. Math. Soc. 38 (1935), 48-88.

[HL1] T. Hu and K. Lau, The sum of Rademacher functions and Hausdorff dimension, Math. Proc. Cambridge Philos. Soc. 108 (1990), 97-103. MR 91d:28020

[HL2] - Hausdorff dimension of the level sets of Rademacher series, Bull. Polish Acad. Sci. Math. 41 (1993), No. 1, 11-18. CMP 96:16

[L1] K. Lau, Fractal measure and mean p-variations, J. Funct. Anal. 108 (1992), No. 2, 427-457. MR 93g:28007

[L2] $\quad$ Dimension of a family of singular Bernoulli convolutions, J. Funct. Anal. 116 (1993), 335-358. MR 95h:28013

[LN1] K. Lau and S. Ngai, Multifractal measure and a weak separation condition, Advances in Math., to appear.

[LN2] - The $L^{q}$-dimension of the Bernoulli convolution associated with the golden number, preprint.

[LP] F. Ledrappier and A. Porzio, A dimension formula for Bernoulli convolutions, J. Statist. Phys. 76 (1994), 1307-1327. MR 95i:58111

[PU] F. Przytycki and M. Urbanski, On the Hausdorff dimension of some fractal sets, Studia Math. 93 (1989), 155-186. MR 90f:28006

[Sa] R. Salem, Algebraic numbers and Fourier analysis, Heath, 1963. MR 28:1169

[Si] C. L. Siegel, Algebraic integers whose conjugates lie in the unit circle, Duke Math. J. 11 (1944), 597-602. MR 6:39b

[So] B. Solomyak, On the random series $\Sigma \pm \lambda^{n}$ (an Erdös problem), Ann. of Math. 142 (1995), 611-625. MR 97d:11125

[St] R. S. Strichartz, Self-similar measure and their Fourier transformations. III, Indiana Univ. Math. J. 42 (1993), 367-411. MR 94j:42025

Department of Mathematics, University of Wisconsin-Green Bay, Green Bay, WisCONSIN 54311

E-mail address: HUT@gbms01.uwgb.edu 